This is the author's final, peer-reviewed manuscript as accepted for publication. The publisher-formatted version may be available through the publisher's web site or your institution's library.

\title{
Environmental rearing effects on impulsivity and reward sensitivity
}

Kimberly Kirkpatrick, Andrew T. Marshall, Jacob Clarke, and Mary E. Cain

\section{How to cite this manuscript}

If you make reference to this version of the manuscript, use the following information:

Kirkpatrick, K., Marshall, A. T., Clarke, J., \& Cain, M. E. (2013). Environmental rearing effects on impulsivity and reward sensitivity. Retrieved from http://krex.ksu.edu

\section{Published Version Information}

Citation: Kirkpatrick, K., Marshall, A. T., Clarke, J., \& Cain, M. E. (2013). Environmental rearing effects on impulsivity and reward sensitivity. Behavioral Neuroscience, 127(5), 712-724.

Copyright: (2013 American Psychological Association

Digital Object Identifier (DOI): doi:10.1037/a0034124

Publisher's Link: http://psycnet.apa.org/journals/bne/127/5/712/

This item was retrieved from the K-State Research Exchange (K-REx), the institutional repository of Kansas State University. K-REx is available at http://krex.ksu.edu 
Environmental rearing effects on impulsivity and reward sensitivity

\author{
Kimberly Kirkpatrick, Andrew T. Marshall, \\ Jacob Clarke, and Mary E. Cain \\ Kansas State University
}

Corresponding author: Kimberly Kirkpatrick, Department of Psychology, 492 Bluemont Hall, 1100 Mid-Campus Drive, Kansas State University, Manhattan, KS 66506-5302. E-mail: kirkpatr@ksu.edu Tel: 785-532-0805. Fax: 785-532-5401.

Running Head: Environmental rearing effects on impulsivity 


\begin{abstract}
Previous research has indicated that rearing in an enriched environment may promote selfcontrol in an impulsive choice task. To further assess the effects of rearing environment on impulsivity, two experiments examined locomotor activity, impulsive action, impulsive choice, and different aspects of reward sensitivity and discrimination. In Experiment 1, rats reared in isolated or enriched conditions were tested on an impulsive choice procedure with a smallersooner versus a larger-later reward, revealing that the isolated rats valued the smaller-sooner reward more than the enriched rats. A subsequent reward challenge was presented in which the delay to the two rewards was the same but the magnitude difference remained. The enriched rats did not choose the larger reward as often as the isolated rats, reflecting poorer reward discrimination. Impulsive action was assessed using a differential-reinforcement-of-low-rate task, which revealed deficits in the enriched rats. In Experiment 2, rats reared in isolated, standard, or enriched conditions were tested on reward contrast and reward magnitude sensitivity procedures. The rats were presented with two levers that delivered different magnitudes of food on variable interval 30-s schedules. Across all tests, the enriched and social rats displayed more generalized responding to the smaller-reward lever, but a similar response to the large-reward lever, compared to the isolated rats. This confirmed the results of Experiment 1, indicating poorer reward discrimination in the enriched condition compared to the isolated condition. The results suggest that enrichment may moderate reward generalization/discrimination processes through alterations in incentive motivational processes.
\end{abstract}

Keywords: Environmental enrichment, isolation rearing, impulsive choice, impulsive action, reward processing 
The differential rearing paradigm typically includes an enriched condition (EC) and an isolated condition (IC; Renner \& Rosenzweig, 1987; Simpson \& Kelly, 2011). EC rats are housed with cohorts in a large cage with novel objects and receive daily handling. In contrast, IC rats are housed individually without novel objects and are not handled during the rearing period (e.g., 30 days). The presence of cohorts, contact with novel objects, and the amount of handling are all critical elements that contribute to the enriched environment (Renner \& Rosenzweig, 1987). These factors singly or collectively alter both the behavior and neurobiology of differentially-reared rats.

Differential rearing alters the response to a variety of drug rewards, including stimulants, opiates, and ethanol (Bardo \& Dwoskin, 2004; Cain, Mersmann, Gill, \& Pittenger, 2012; Coolon \& Cain, 2009; Deehan, Cain, \& Kiefer, 2007; Deehan, Palmatier, Cain, \& Kiefer, 2011; T. A. Green, Gehrke, \& Bardo, 2002; J. K. Smith, Neill, \& Costall, 1997; M. A. Smith, Bryant, \& McClean, 2003; M. A. Smith et al., 2005; Stairs \& Bardo, 2009), and it has been suggested that the effects are due to altered sensitivity to drug rewards. Differences in rearing environment also affect sensitivity to non-drug rewards such as locomotor activity in a novel environment (Bowling, Rowlett, \& Bardo, 1993; Lore \& Levowitz, 1966), responses to novel stimuli (Cain, Green, \& Bardo, 2006; Zimmermann, Stauffacher, Langhans, \& Würbel, 2001), and responses to sucrose (Brenes, Padilla, \& Fornaguera, 2009; Gill \& Cain, 2010).

In addition to differences in reward sensitivity, there is also some indication that differential rearing may produce differences in impulsive behaviors. Previous research has suggested that impulsivity is a multidimensional construct that can be divided into two overarching subtypes -- impulsive choice and impulsive action (Diergaarde et al., 2008). Impulsive choice results in preference for smaller, more immediate versus larger, more delayed 
outcomes, while impulsive action refers to an inability to inhibit motor responses (Brunner \& Hen, 1997; Diergaarde, et al., 2008; Evenden, 1999; also see Ho, Al-Zahrani, Al-Ruwaitea, Bradshaw, \& Szabadi, 1998).

Currently, there is no consensus concerning whether EC or IC rats are more impulsive than their counterparts. With regard to impulsive action, IC rats have been reported to display decreased premature responding during a rat gambling task (Zeeb, Wong, \& Winstanley, 2012), and while under the influence of amphetamine in a five-choice serial reaction time task (Dalley, Theobald, Pereira, Li, \& Robbins, 2002) in comparison to socially housed rats. However, in a fixed minimum interval task, which is a variant of the differential-reinforcement-of-low-rate (DRL) schedule, IC rats were also reported to display fewer impulsive responses than socially housed rats (Hill, Covarrubias, Terry, \& Sanabria, 2012), but on a DRL task IC rats displayed difficulty in inhibiting responses (Ough, Beatty, \& Khalili, 1972). A similarly mixed pattern of results has been disclosed in impulsive choice tasks, evaluating choices between a smallersooner (SS) reward versus a larger later (LL) reward. Perry et al. (2008) found that IC rats preferred the SS reward more than EC rats did, suggestive of increased impulsive choice. However, Hellemans et al. (2005) found that EC rats were more impulsive than IC rats in a similar choice task. Given such a discrepancy in results regarding impulsivity in differentiallyreared animals, as well as the possible independence of impulsivity in impulsive action versus impulsive choice tasks (see Evenden, 1999), any previous conclusions concerning greater impulsivity in IC (or EC) rats may have provided only a partial account of the effects of differential rearing on impulsivity.

The picture from the previous literature is complex and inconsistent. Isolated rearing appears to increase locomotor activity in novel environments, but predominately decrease 
impulsive action, while possibly increasing impulsive choice. However, the results of the impulsive action and impulsive choice studies are conflicting, with some studies showing the opposite to the predominate pattern. None of the previous studies have attempted to examine all three of these key constructs within the same experimental context. As a result, differences in rearing practices and experimental methodologies could underlie the conflicting patterns in the literature. In an effort to pin down these patterns and examine possible mechanisms behind the effects of rearing environment on locomotor activity and the different types of impulsivity, the present pair of studies examined measures of impulsive action, impulsive choice, locomotor activity (Experiment 1) and amphetamine-induced locomotor activity (Experiment 2). In addition, tests of reward sensitivity (Experiment 2) and reward discrimination (Experiments 1 and 2) were included to determine whether a common mechanism may underlie the three behavioral patterns, and whether differences in activity and impulsive behaviors may relate to differences in reward sensitivity as a function of rearing environment.

\section{Experiment 1}

\section{Method}

\section{Animals}

Eighteen experimentally-naïve male Sprague-Dawley rats (Charles River; Portage, MI) arrived at the facility (Kansas State University; Manhattan, KS) at 21 days of age. Their housing conditions varied according to rearing condition (see Procedure - Environmental rearing). Prior to the onset of operant behavioral testing, the rats were placed on a restricted diet consisting of $15 \mathrm{~g}$ of lab chow per day. During operant behavioral testing, the rats received approximately half of their daily ration in the form of 45-mg pellets (TestDiet MLab; Richmond, IN), delivered as reinforcers in the experimental chambers. The rats had ad libitum access to water in both the 
home cages and experimental chambers. The colony room was maintained on a 12:12 hr light:dark cycle with lights on at approximately 6 a.m.

\section{Apparatus}

Locomotor behavior was measured in a locomotor chamber, measuring $41 \times 41 \times 41 \mathrm{~cm}$ (Coulbourn Instruments; Whitehall, PA). The chamber consisted of Plexiglas walls and plastic flooring covered with bedding. A photobeam sensor ring consisted of a $16 \times 16$ horizontal photocell array spaced $2.5 \mathrm{~cm}$ apart (TruScan 2.01; Coulbourn Instruments) and was linked to a

personal computer located outside of the chambers. A white-noise generator ( $70 \mathrm{~dB})$ was used to create ambient background noise to mask sounds from outside the chamber.

The impulsive action and impulsive choice procedures were conducted in 18 operant chambers (Med Associates; St. Albans, VT). Each chamber $(25 \times 30 \times 30 \mathrm{~cm})$ was housed inside of a ventilated, noise-attenuating box $(74 \times 38 \times 60 \mathrm{~cm})$, and was equipped with a stainless steel grid floor, two stainless steel walls (front and back) and a transparent polycarbonate sidewall, ceiling, and door. A magazine pellet dispenser (ENV-203) delivered 45mg food pellets (TestDiet MLab; Richmond, IN) into the food cup (ENV-200R7), which was centered on the lower section of the front wall. Each head entry into the food cup was transduced by an LED-photocell (ENV-254). Two retractable levers (ENV-112CM) were situated on either side of the food cup at approximately one third of the total height of the chamber. Directly above each lever was a nose poke key which could be illuminated by a cue light (ENV-119M-1). Water was available ad libitum from a water bottle mounted outside the chamber that included a metal tube that protruded through a hole in the lower-center of the back wall. Experimental events were controlled and record with 2-ms resolution by the software program MED-PC IV (Tatham \& Zurn, 1989). 


\section{Procedure}

Environmental rearing. The rats were housed in pairs in standard plastic shoebox cages for two days prior to an initial locomotor activity test. Following the first assessment of the rats' locomotor behavior (see Locomotor activity), the rats were partitioned into two groups and subjected to differential rearing in either an enriched (EC) or isolated (IC) condition ( $n=9)$, with group assignments matched according to initial locomotor activity levels. The rats were housed in these conditions for the duration of the study. EC rats were housed together in a large metal cage $(60 \times 120 \times 45 \mathrm{~cm})$ with pulp paper bedding, which contained 14 novel objects (e.g., PVC pipe, buckets, children's toys, etc.). Each day, the EC rats were handled and half of the objects were replaced with new objects; the remaining items were rearranged into a novel configuration. Between one and two times per week, all objects were replaced with new items. IC rats were housed individually in hanging wire cages with a wire mesh floor and front panel $(17 \times 24 \times 20$ cm), and solid metal sides, back and top. IC rats were not handled during the rearing period (2353 days of age). These specific rearing environments have been employed extensively in previous studies (Renner \& Rosenzweig, 1987; Simpson \& Kelly, 2011). As the goal of the present set of experiments was to compare these rearing environments with the key reference literature on impulsive behaviors in rats, as opposed to isolating the source of the corresponding behavioral differences (e.g., group housing, the presence of novel objects, handling), only these well-established rearing conditions were employed here.

Locomotor activity. To measure locomotor behavior, the rats were placed in the locomotor chambers for $90 \mathrm{~min}$ and the total distance traveled (m) was recorded. This test was conducted on the second day after arrival and then again on the day following the 30-day rearing period. During the first test, the floor was covered with pine chip bedding; to maintain the 
novelty of the locomotor chambers, the floors were covered with corn cob bedding and the Plexiglas walls were covered with opaque plastic sheets during the second test.

Impulsive choice. The impulsive choice procedure consisted of three phases - an impulsive choice task (Phase 1) and a reward challenge task (Phases 2-3). The impulsive choice task was adapted from Green and Estle (2003); this procedure has been used previously in our laboratory and has been shown to be sensitive in detecting stable individual differences (Galtress, Garcia, \& Kirkpatrick, 2012), as well as strain differences (Garcia \& Kirkpatrick, 2013) in choice behavior in rats. The rats received a choice in which either a smaller outcome was delivered after a short period of time (i.e., smaller-sooner, SS) or a larger outcome that was delivered after a longer period of time (i.e., larger-later, LL) outcome. An SS choice resulted in delivery of a one-pellet reward after a 10-s delay, whereas an LL choice resulted in the delivery of a two-pellet reward after a 30-s delay. Each of the two levers corresponded to either the SS or LL outcome; the lever-choice configurations were counterbalanced across rats.

Half of the rats were tested in the impulsive choice task first, whereas the other half were tested following the impulsive action task. Within each session, there were three different trial types: forced choice, free choice, and peak trials. During forced choice trials, one lever corresponding to either the SS or LL outcome was inserted into the chamber. A response on that lever resulted in the onset of the cue light above the lever. After the target delay elapsed (i.e., 10 s or $30 \mathrm{~s}$ ), food became available to be delivered; the next response on that lever terminated the trial, resulting in lever retraction, cue light offset, food delivery (i.e., one or two pellets), and a 60-s intertrial interval (ITI). Free choice trials began by inserting both levers. Following the first response on either lever (i.e., the choice), the alternative lever was retracted and the cue light above the chosen lever was illuminated. Following the first response after the target delay had 
elapsed (i.e., 10 s or 30 s), the trial terminated, resulting in lever retraction, cue light offset, food delivery, and the 60-s ITI. A fixed ITI was selected because it more closely mimics real-life choice situations by allowing for long-term reward maximization (Odum, 2011). On peak trials (Roberts, 1981), one lever was inserted into the chamber. As with forced choice trials, the first response on this lever resulted in the illumination of the light and initiation of a 90-s interval. At the end of the interval, the light was terminated and the 60-s ITI began; no food was delivered during peak trials. Training continued until the choice behavior met a stability criterion of 3 consecutive sessions with less than 10\% deviation from the mean, which required 21 and 20 sessions for the rats that received the impulsive choice task first and second, respectively.

The reward challenge task was identical to the impulsive choice task except that the delay to the one-pellet SS outcome was extended to 30 s. In Phase 2, the lever-choice configurations for each individual rat were identical to those in the impulsive choice task; in Phase 3, the leverchoice configurations were switched (i.e., if the left lever corresponded to the SS outcome in Phases 1-2, then the left lever corresponded to the LL outcome in Phase 3). Training continued until the choice behavior met the stability requirement, which required 13 and 27 sessions for Phases 2 and 3, respectively, for the rats that were trained on the impulsive choice task first. For the remaining rats, Phases 2 and 3 lasted for 13 and 23 sessions, respectively.

Each session lasted for approximately $13 \mathrm{hr}$ and consisted of four blocks of trials separated by a 90-min inter-block interval (IBI). Each trial block consisted of 8 SS forced choice, 2 SS peak, 8 LL forced choice, 2 LL peak, and 30 free choice trials.

Impulsive action. The impulsive action procedure consisted of a DRL schedule (Catania, 1970). A block of trials began by illuminating either the left or right cue light to indicate activation of the nose poke key. In each block of trials, the first nose poke response initiated the 
schedule of reinforcement. Food delivery on the DRL schedule of reinforcement was contingent on the rat spacing its consecutive responses by at least $t \mathrm{~s}$. If the rat responded before $t \mathrm{~s}$ had elapsed, then the interval reset and the rat was required to wait for at least another $t \mathrm{~s}$ before a response resulted in food delivery. Each block of training lasted until a total of 80 food deliveries occurred. The IBI was $180 \mathrm{~min}$, and a maximum of 3 blocks were delivered in each session. The criterion value $(t)$ was $30 \mathrm{~s}$ and then $60 \mathrm{~s}$ in consecutive phases. For the rats that experienced the impulsive action task first, the DRL 30-s phase lasted for 21 sessions, and the DRL 60-s phase lasted for 40 sessions to achieve stable performance. For the remaining rats, the DRL 30-s and DRL 60-s phases lasted for 20 and 36 sessions, respectively. Each session lasted approximately $13 \mathrm{hr}$.

\section{Data Analysis}

Locomotor activity. The primary dependent measure was the total distance moved (m). The infrared-beam array tracked the location of the rat in consecutive 0.5-s intervals across the test session; the location of the rat was defined as the center of all beams crossed in the .5-s interval. Total distance moved was derived from the change in location across the test session. The locomotor testing results were subjected to a $2 \times 2$ repeated measures analysis of variance (ANOVA) with rearing condition (EC, IC) as the between-subjects variable and pre/post (prerearing, post-rearing) as the within subjects variable. Tukey post-hoc tests were used to identify the source of significant interactions here and throughout.

Impulsive choice. Two dependent measures were used in the impulsive choice analyses. One was the proportion of choices for the LL outcome. This was calculated as the number of choices for the LL outcome divided by the total number of choices. For the impulsive choice task, the final ten sessions of Phase 1 were included in the analysis. For the reward challenge 
task, the final ten sessions of Phases 2 and 3 were collapsed to compute a single mean choice LL measure for each rat, as there were no significant differences between the two reward challenge phases. The proportion of choices for the LL outcome in both phases was compared across rearing conditions in each phase using an independent-samples $t$-test.

The second dependent measure was the mean latency $\left(\log _{10} \mathrm{~s}\right)$ to initiate forced choice trials over the final ten sessions of each phase. Previous research has indicated that the latency to begin forced choice trials is an index of outcome value that predicts choice behavior in starlings and rats (Bateson \& Kacelnik, 1995; Kacelnik, Vasconcelos, Monteiro, \& Aw, 2011; Reboreda \& Kacelnik, 1991; Roesch, Calu, \& Schoenbaum, 2007; Shapiro, Siller, \& Kacelnik, 2008). Latency was measured as the time between the insertion of the lever on a forced choice trial and the first response on that lever (i.e., the onset of the forced-choice delay). Due to the presence of long latencies, the latencies were transformed to a $\log _{10}$ scale. For the impulsive choice and reward challenge tasks, the latencies were subjected to a $2 \times 2$ repeated-measures ANOVA with trial type (SS, LL) as the within-subjects factor and rearing condition (IC, EC) as the betweensubjects factor. Additionally, the difference in latencies to initiate SS and LL forced choice trials was used as an index of discrimination between the two outcomes; the greater the difference, the greater the discrimination and the stronger the preference for one outcome over the other. For each rat, the mean forced choice LL latency was subtracted from the mean forced choice SS latency. Analyses were also conducted to determine if these scores differed significantly from zero. Subsequently, the latency difference measure for each phase was compared across rearing conditions using an independent samples $t$-test.

The peak trials were included in the procedure to examine timing behavior as an index of learning the delay to rewards. These were subjected to analyses, which confirmed that both 
rearing conditions learned the durations and expressed normal timing. Because there were no rearing differences, and because these analyses were not central to the theme of the paper, the data are not reported.

Impulsive action. The final ten sessions of each DRL phase were used for analysis. Two rats, one from each rearing condition, were removed from analysis of the DRL procedure due to equipment problems. The primary dependent measure was the number of nose poke key responses for every reward delivered, a measure of performance efficiency on the DRL task (i.e., the lower the number of responses/reward, the more efficient the performance). Responses per reward was calculated as the total number of responses on the nose poke key during the DRL task divided by the total number of rewards received. The responses/reward measure was analyzed using a $2 \times 2$ repeated-measures ANOVA, with rearing condition (EC, IC) as the between-subjects factor and DRL criterion (DRL 30, DRL 60) as the within-subjects factor.

Statistical analyses. All statistical analyses were conducted in SPSS. The $\alpha$-value for reporting of significant results was set at $p<.05$.

\section{Results and Discussion}

\section{Locomotor Activity}

Figure 1 shows the total distance moved by the IC and EC rats prior to (pre) and following (post) the 30-day rearing period. The IC rats showed a large increase in the total distance moved from the pre- to post-rearing locomotor tests, while the EC rats showed a decrease in total distance moved across the same time frame. An ANOVA revealed a main effect of pre/post, $F(1,16)=8.22, p=.011$, a main effect of rearing condition, $F(1,16)=10.40$, $p=.005$, and a Pre/Post $\times$ Rearing Condition interaction, $F(1,16)=39.14, p<.001$. Tukey post-hoc tests revealed that the EC and IC groups did not differ in the pre-rearing locomotor test, 
but that IC rats showed significantly greater locomotor activity than EC rats in the post-rearing locomotor test, $p<.05$. Therefore, as previously reported (Fone \& Porkess, 2008; Simpson \& Kelly, 2011), locomotor activity was lower in EC rats compared to IC rats following rearing.

\section{Impulsivity}

Impulsive choice. Figure 2 shows the proportion of choices for the LL outcome in the impulsive choice and the reward challenge phases. In the impulsive choice task, the IC and EC rats did not differ in their proportion of choices for the LL outcome, $t(16)=0.74, p=.469$. However, during the reward challenge task, in which the delay to both outcomes was $30 \mathrm{~s}$, the IC rats chose the LL outcome significantly more than the EC rats did, $t(16)=2.76, p=.014$. Therefore, the IC rats were sensitive to the difference in reward magnitude between the onepellet and two-pellet outcomes, suggesting that differential rearing may have altered reward sensitivity (see Brenes \& Fornaguera, 2008).

To gain an additional assessment of the value of the SS and LL outcomes, Figure 3 (top panel) shows the mean latency to initiate the SS and LL forced choice trials. During the impulsive choice task, the EC rats showed similar latencies to initiate the SS and LL forced choice trials, while the IC rats showed longer latencies to initiate the LL forced choice trials than they did for the SS forced choice trials. During the reward challenge task, both the EC and IC rats showed shorter latencies to initiate the LL forced choice trials than they did the SS forced choice trials (Figure 3). For both the impulsive choice and reward challenge tasks, there was a main effect of trial type, $F s(1,16)>5.7, p s<.05$, and a Trial Type $\times$ Rearing Condition interaction, $F \mathrm{~s}(1,16)>5.2$, $p \mathrm{~s}<.05$, but no significant main effect of rearing condition, $F \mathrm{~s}(1$, 16) $<1$. Tukey post-hoc tests revealed that IC rats showed significantly longer LL latencies in the impulsive choice task relative to EC rats, but the SS latencies were not different. The shorter 
LL latencies by the EC rats in the impulsive choice task suggests that the EC rats valued the LL outcome more than the IC rats did, especially given the similarities in the SS latencies between the EC and IC rats. In the reward challenge task there was a near-significant difference in latencies for the LL choice, with the IC rats displaying shorter LL choice latencies, but there was no difference in SS latencies. In the reward challenge task, both groups displayed shorter LL than SS latencies, but the difference in SS versus LL latency appeared larger in the IC rats.

To provide a more concrete analysis of within-group differences in latencies to respond on SS and LL forced choice trials, Figure 3 (bottom panel) shows the mean difference score (i.e., SS $\log _{10}$ latency - LL $\log _{10}$ latency) for both the impulsive choice and reward challenge tasks. The IC rats showed a greater (i.e., more negative and more positive) difference score than the EC rats did in the impulsive choice and the reward challenge task, respectively. With the exception of the impulsive choice task difference score for the EC rats, $t(8)=0.06, p=.95$, all differences scores were significantly different from zero. The impulsive choice task difference score for the IC rats was significantly less than zero, $t(8)=4.09, p=.003$, and the reward challenge task difference score for both the EC and IC rats was significantly greater than zero, ts(8) > 3.51, ps < .01. Independent-samples $t$-tests revealed that the IC rats showed a significantly more negative difference score than the EC rats did in the impulsive choice task, $t(16)=2.30, p=.035$. This indicates that the IC rats valued the LL outcome less than the SS outcome, whereas the EC rats valued the SS and LL outcomes more similarly. In the reward challenge task, the IC rats showed a significantly more positive difference in latencies than the EC rats did, $t(16)=2.29, p=.036$, indicating that the IC rats valued the LL outcome more than the SS outcome, and to a greater extent than the EC rats. 
Overall, although there were no detectable differences between the EC and IC rats in the proportion of choices for the LL outcome, the latency results from the impulsive choice task suggested that the IC rats valued the LL to a lesser degree than the EC rats, confirming previous results showing more impulsive behavior in choice tasks in IC rats (Perry, et al., 2008). Given that enrichment enhances DA functioning within the mPFC (Zhu, Apparsundaram, Bardo, \& Dwoskin, 2005), these findings are consistent with the recent results suggesting that decreasing DA function within the mPFC increases impulsive choice (Pardey, Kumar, Goodchild, \& Cornish, 2012). Additionally, as EC rats have shown elevated 5HT levels in the PFC relative to socially isolated rats (Brenes, Rodríguez, \& Fornaguera, 2008), the present results also seem consistent with results suggesting a negative relationship between serotonergic transmission in the mPFC and the degree of impulsive choice behavior (Barbelivien, Billy, Lazarus, Kelche, \& Majchrzak, 2008). Therefore, it is plausible that enriched rats display a decrease in impulsive choice due to enhanced DA and 5HT function, relative to IC rats. In the reward challenge task, the delays to both the SS and LL outcomes were identical so that reward magnitude alone should determine choice behavior. Here, the IC rats were significantly more likely to choose the larger outcome than the EC rats. Concerning the forced choice latencies, the IC rats showed a greater difference between the SS and LL trials than the EC rats in both the impulsive choice and reward challenge tasks. Both sets of results suggest that the IC rats were more sensitive to the differences in reward magnitude than the EC rats. The results of the reward challenge task are consistent with a recent report indicating that IC rats were driven to choose the larger magnitude, even when it was disadvantageous to do so (Zeeb, et al., 2012). In the present task, choosing the larger magnitude was advantageous, but it is possible that they nonetheless reflect a stronger preference for larger magnitudes in the absence of differences in delay. This preference in the IC 
rats appeared to be overridden in the impulsive choice task, where the IC rats displayed higher valuation of the SS option, most likely due to the allure of the shorter delay to reward which was associated with a higher local rate of reward (1 pellet in $10 \mathrm{~s}$, as opposed to 2 pellets in $30 \mathrm{~s}$ ).

Impulsive action. Figure 4 shows the number of responses per reward received during the DRL task for inter-response time (IRT) criteria of 30 and 60 s. An ANOVA revealed that IC rats made significantly fewer responses per reward than EC rats, $F(1,14)=6.59, p=.022$. There also was a DRL Criterion $\times$ Rearing Condition interaction, $F(1,14)=6.00, p=.028$, but no main effect of DRL criterion, $F(1,14)=1.88, p=.191$. Tukey post-hoc tests revealed that IC rats made significantly fewer responses per reward than EC rats when the DRL criterion was 30 s, but not when the DRL criterion was $60 \mathrm{~s}$.

Although the IC rats appeared to be more impulsive in the choice task, the EC rats were more impulsive in the impulsive action task, similar to previous results (Dalley, et al., 2002; Hill, et al., 2012; Zeeb, et al., 2012). A possible mechanism that could explain differences in impulsive choice and impulsive action is a difference in reward sensitivity, which has been well documented to emerge as a function of differential rearing. The results from the reward challenge task described above support such an explanation. Assuming that differential rearing does produce differential sensitivity to reward magnitude, the apparent contradiction of the impulsive choice and impulsive action results, as well as those of previous studies, may be reconcilable. As previously discussed, the serotonergic system has been extensively implicated in impulsive behaviors (e.g., Ho, et al., 1998). However, previous research has also suggested the involvement of the serotonergic system in processes related to reward sensitivity (Bari et al., 2010). Accordingly, the differences in impulsivity in IC rats, relative to EC rats, may reflect an increased sensitivity to reinforcement parameters in the IC rats; the IC rats were more efficient in 
the DRL procedure and earned more reward in the reward challenge task, but valued shorter delays to reward (associated with a higher local rate of reinforcement) in the impulsive choice task. Therefore, the goal of Experiment 2 was to further investigate the potential rearing environment-induced differences in sensitivity to different reward magnitudes. In addition, because Experiment 1 only assessed the extremities of the commonly used rearing condition paradigms, Experiment 2 added a standard, pair-housed condition (SC) to assess whether the effects of rearing environment were due more predominantly to environmental enrichment factors (e.g., exposure to novelty) or social isolation factors, as previous work has shown that social isolation can produce profound effects on brain and behavioral processes (see Fone \& Porkess, 2008; Renner \& Rosenzweig, 1987) that may differ from the effects of environmental enrichment (e.g., Renner \& Rosenzweig, 1987; Simpson \& Kelly, 2011).

\section{Experiment 2}

\section{Method}

\section{Animals}

Twenty-four experimentally-naïve male Sprague-Dawley rats (Charles River; Portage, MI) arrived at the facility (Kansas State University; Manhattan, KS) at 21 days of age. Their housing conditions varied according to experimental condition (see Environmental rearing). Prior to the onset of behavioral testing, the rats were placed on a restricted diet to maintain their weight at a target of $85 \%$ of free-feeding weight, determined by growth curves obtained from the supplier. During behavioral testing, the rats received part of their daily ration in the form of 45mg pellets (BioServ), delivered as reinforcers in the experimental chambers and the remaining ration delivered in the colony room. The rats had free access to water at all times, in both the home cages and experimental chambers. The colony room was maintained on a 12:12 hr 
reversed light:dark cycle with lights off at 8 a.m. Several red lamps provide illumination throughout the dark cycle.

\section{Apparatus}

The apparatuses were the same as in Experiment 1 for both the locomotor activity and the reward sensitivity testing.

\section{Procedure}

Environmental rearing. Twenty-four rats were housed in pairs in standard plastic shoebox cages for two days prior to an initial locomotor activity test (see Locomotor activity). Then, the rats were partitioned into groups and subjected to differential rearing in an EC, SC, or IC rearing environment ( $n=8)$, with group assignments matched according to initial locomotoractivity levels. The rats were housed in these conditions for the duration of the study. The conditions for the EC and IC rats were the same as in Experiment 1. The SC rats were housed in pairs in standard shoebox cages and were handled daily. They did not receive any other form of enrichment.

Locomotor activity. For assessments of locomotor activity in a novel environment, rats were placed in the locomotor chambers for $60 \mathrm{~min}$ and the total distance traveled was recorded as in Experiment 1. There were four assessments of locomotor activity conducted on the following days: Test 1 - Day 2 after arrival (at age 23 days); Test 2 - following the 30-day rearing period (at age 54 days); Tests 3 and 4 - after the reward sensitivity testing to assess the effects of d-amphetamine or saline. For Tests 3 and 4, half of the rats in each rearing condition were injected with $0.3 \mathrm{mg} / \mathrm{kg}$ of d-amphetamine and the other half were injected with sterile saline (vehicle) immediately prior to insertion in the locomotor chambers. The order of drug/saline receipt was counterbalanced across rats. There was a single rest day between Test 3 
and Test 4 to allow for wash out of the drug. To maintain the novelty of the locomotor chambers different types of bedding and wall patterns were used in each test.

Reward sensitivity procedure. The reward sensitivity procedure involved a discretetrial VI 30-s schedule delivered on two different levers. The onset of a trial was cued by inserting one of the levers, which initiated the VI schedule. The first response after the VI $30 \mathrm{~s}$ timed out was rewarded with one or more food pellets and then the lever was retracted. There was a variable ITI of 120 s prior to the onset of the next trial. The probability of a left or right trial was .5 and was determined pseudo-randomly with the constraint of no more than two trials in a row on the same side. Sessions lasted for $2 \mathrm{hr}$ and terminated at the end of a trial. Each phase of testing lasted for 10 sessions. Prior to continuing to the next phase, checks were conducted for stability in responses rate on each lever by assessing individual-session deviations from the mean over the last three days of training. In all cases, deviations were within $\pm 20 \%$ of the mean.

Baseline. All rats initially received 1 food pellet on each lever to establish baseline response rates.

Reward contrast sensitivity. During the reward contrast sensitivity phase, the rats received 4 food pellets on one of the levers (LG-4), with the lever assignment counterbalanced across rats. This allowed for an assessment of induction (increased response on the 4-pellet induction lever, LG-4) and negative contrast (decreased response on the unchanged 1-pellet contrast lever, SM-1).

Reward magnitude sensitivity. During the reward magnitude sensitivity phase, the rats received different numbers of pellets on each lever. This assessed sensitivity to changes in reward magnitude across phases and to differences in reward magnitude within a phase. The 
large reward remained on the same side throughout the test and this was the same as the large reward lever in the contrast sensitivity phase. The four treatments during this phase were: 1 versus 2 pellets; 1 versus 3 pellets; 2 versus 3 pellets; and 2 versus 4 pellets, delivered in either an ascending or descending order (counterbalanced across rats). Each pair of magnitudes was delivered for 10 sessions, after which the rats were returned to the baseline training conditions.

\section{Data Analysis}

Locomotor activity. The locomotor activity tests were analyzed in the same general fashion as in Experiment 1. Separate analyses were conducted for the pre/post rearing tests and the subsequent saline (SAL) versus amphetamine (AMP) tests using $2 \times 2$ ANOVAs with the variables of rearing condition and pre/post (or SAL/AMP). A bivariate correlation coefficient was additionally computed to compare the post-rearing activity scores with the post-rearing SAL test to determine the long-term stability in the locomotor behavior.

Reward sensitivity. For all phases of the reward sensitivity test, the mean response rate on each of the levers was determined by dividing the number of responses during a trial by the VI duration on that trial, and then averaging across trials. The analysis was restricted to include only the first $30 \mathrm{~s}$ of VIs lasting longer than $30 \mathrm{~s}$ (the mean of the VI schedule) to avoid any reductions in rate that may occur with long intervals. For any VI less than $30 \mathrm{~s}$, the entire trial was included. In addition, the response rate average only included the first half of each session. This was due to the observation of satiety effects on response rates during the latter half of the session in the higher reward magnitude conditions. For example, in the reward contrast phase, there was a significant reduction in responding in the second half of the session, $F(1,21)=39.3$, $p<.001$. However, there were no differences in rates of satiety in the different rearing 
conditions, $F(2,21)=1.9, p=.176$. The same general patterns were observed in all of the reward sensitivity phases.

Because the baseline response rates were higher for the IC rats, compared to the EC and SC rats, analyses on subsequent phases utilized a ratio change score which was the response rate during each subsequent phase divided by the initial baseline response rate. A ratio of 1.0 would indicate no change from baseline, whereas ratios less than 1.0 were indicative of rate decreases and greater than 1.0 of rate increases. For the baseline and reward contrast sensitivity phases, the response rates on each lever (or relative rates on each lever) were subjected to an ANOVA with the variables of lever (small vs. large) and rearing condition (IC, SC, or EC). For the reward magnitude sensitivity phases, separate analyses were conducted on the relative response rates on small and large levers with the variables of reward magnitude and rearing condition. Tukey post-hoc tests were conducted on significant interactions in all cases.

Statistical analyses. All statistical analyses were conducted in SPSS. The $\alpha$-value for reporting of significant results was set at $p<.05$.

\section{Results and Discussion}

\section{Locomotor Activity}

Figure 5 displays the total distance moved as a function of rearing condition during the pre-rearing (pre) and post-rearing (post) tests as well as during the SAL and AMP tests conducted after completion of the reward sensitivity task (approximately eight months after rearing). A comparison of the pre- and post-rearing activity data indicated an effect of rearing condition, $F(2,21)=6.39, p=.007$, and a Pre/Post $\times$ Rearing Condition interaction, $F(2,21)=$ $5.14, p=.015$, but no main effect of pre/post, $F(1,21)=2.63, p=.120$. Follow up analyses on the interaction indicated a significant rearing condition effect during the post-rearing period, $F(2$, 
21) $=9.48, p=.001$, which was due to greater activity in the IC and SC rats compared to the EC rats. There was no effect of rearing condition in the pre-rearing test, $F(2,21)=1.28, p=.299$. These findings replicate the locomotor results of Experiment 1, and extend on those findings by indicating that the SC and IC rats were more similarly affected by rearing conditions (Cain, et al., 2012; Wooters et al., 2011).

A separate analysis was conducted to assess the effect of AMP versus SAL on locomotor activity. As seen Figure 5, AMP produce a substantial increase in locomotor activity, $F(1,21)$, = 79.30, $p<.001$. The rearing condition effects were evident under both SAL and AMP, $F(2,21)$ $=6.07, p=.008$, and there was no SAL/AMP $\times$ Rearing Condition interaction, $F(2,21)=2.31, p$ $=.124$. The results of the AMP test concur with previous research by indicating that EC rats had lower locomotor activity levels, even when under the influence of AMP (Bardo et al., 1995; Bardo \& Dwoskin, 2004; Cain, et al., 2012).

To assess the stability of the rearing effects on locomotor activity over the course of the study, bivariate correlations were computed including the pre- and post-rearing activity scores as well as the saline and amphetamine tests, including all rats in the analysis. There was a substantial correlation between the post-rearing activity scores and the activity under both SAL, $r$ $=.81, p<.001$, and AMP, $r=.62, p=.001$, and between the SAL and AMP activity scores $(r=$ $.62, p=.001)$, but there was no correlation between the pre-rearing activity scores and any of the post-rearing tests, largest $r=.29$. The significant correlation between the post-rearing and saline activity scores indicates that the effects of environmental rearing were maintained over the course of the study. The lack of correlation between the pre- and post-rearing tests indicates that the post-rearing locomotor results were primarily determined by the rearing condition rather than by any pre-existing differences in locomotor activity. 


\section{Reward Sensitivity}

Baseline. The baseline response rates during the initial phase in which there was a 1pellet reward on each lever were examined as a function of rearing condition to determine whether there were any pre-existing differences in the rearing conditions and also to assess whether there were any differences in responding on the small- and large-reward levers. The response rates are shown in Figure 6. As seen in the figure, the IC rats displayed substantially higher response rates on both levers compared to the SC and EC rats. There did not appear to be any differences between the levers. There was a significant main effect of rearing condition, $F(2,21)=9.00, p=.002$, but no effect of lever, $F(1,21)=1.31, p=.265$, and no Lever $\times$ Rearing Condition interaction, $F(2,21)<1$. The rearing condition main effect was due to higher response rates in the IC rats than in the EC and SC rats, which did not differ. The higher rates of responding by the IC rats is consistent with their greater locomotor activity, but the SC rats did not show similarly high response rates in the baseline phase even though they had displayed higher locomotor activity scores. This suggests that an additional process may have driven the IC rats' response rates to higher levels in the baseline phase. One factor that may be important is incentive motivation to work for reward, which may have been enhanced in the IC rats. This issue will be discussed further in the General Discussion.

Reward contrast sensitivity. Reward contrast effects were assessed by increasing the magnitude on the large lever from 1 to 4 pellet(s), while holding the small magnitude constant at 1 pellet. The results from this test are shown in Figure 7 for the three rearing conditions. Due to the pre-existing differences in baseline rates, the data are expressed as a relative response rate measure (see Data Analysis), which is a change from baseline, with a relative rate of 1.0 indicating no change. As seen in the figure, all three rearing conditions showed an increase in 
responding on the large lever. There was no indication of a negative contrast effect on the small lever in that the response rates on that lever did not decrease below 1.0 (no change). An ANOVA revealed a significant lever effect, $F(1,21)=6.85, p=.016$, but no effect of rearing condition, $F(2,21)<1$, or any interaction, $F(2,21)<1$. As an additional assessment of reward induction due to the increase in magnitude, the relative rates for each rearing condition were entered into a one-sample $t$-test, with a comparison value of 1.0 (no change). This revealed significant induction in all three rearing conditions, smallest $t(7)=2.81, p=.026$. Similarly, the relative rates on the small lever were compared to 1.0 to assess negative contrast in each rearing condition. These comparisons did not reveal any significant differences, largest $t(7)=1.30, p=$ .234 .

An additional analysis was conducted on the absolute response rates during the contrast sensitivity test (data not shown) to determine whether there were any differences in the rearing conditions in their discrimination of the large versus small rewards. This revealed no difference in the response rate to the large versus small reward in the EC rats, $t(7)=1.41, p=.201$, or the SC rats, $t(7)=1.44, p=.192$, but the IC rats did significantly discriminate between the large and small rewards, $t(7)=2.93, p=.022$.

The results from the reward contrast sensitivity phase indicate that there were no differences in the induction response to an increase in reward magnitude, but that the IC rats generalized less to the small lever than the EC and SC rats. These results are consistent with Experiment 1, which indicated enhanced reward discrimination in the IC rats. Greater reward discrimination could emerge from increased sensitivity to rewards and reward-related stimuli and increased incentive motivational processes that have been previously reported in socially-isolated rats (Beckmann \& Bardo, 2012; Brenes \& Fornaguera, 2008; Jones, Marsden, \& Robbins, 1990; 
Morgan \& Einon, 1975; Wood, Siegel, \& Rebec, 2006). If the IC rats are more sensitive to rewards, then they would presumably be more selective in their response to rewards of differing magnitudes. This also indicates that the IC rats are not universally more responsive than the EC and SC rats, which suggests that motor hyperactivity is not a likely mechanism for the rearing condition effects on behavior.

Reward magnitude sensitivity. Reward magnitude sensitivity was assessed separately for the small and large levers to determine the effect of changes in reward magnitude on each lever on the relative rate of responding. The results for the large lever are displayed in Figure 8, which are collapsed across the large magnitudes of 2 ( 1 vs. 2), 3 (1 vs. 3 and 2 vs. 3) and 4 (2 vs. 4) pellets for each rearing condition. As seen in the figure, all three rearing conditions showed increases in responding to the large rewards. This was most evident in the SC rats. An ANOVA revealed significant effect of reward magnitude, $F(2,42)=8.77, p=.001$, and a near-significant effect of rearing condition, $F(2,21)=3.38, p=.053$. There was no significant interaction, however, $F(4,42)<1$. The near-significant rearing condition effect was due to greater relative response rates in the SC rats compared to the IC rats, with intermediate relative response rates in the EC rats.

An additional analysis was conducted on the relative rates using a one-sample t-test to compare the relative rates for each large magnitude in each group against no change (1.0). This revealed a significant increase in relative response rate in all three groups to all three magnitudes, smallest $t(7)=2.58, p=.036$.

The results for the small lever are displayed in Figure 9, which are collapsed across the small magnitudes of 1 ( 1 vs. 2 and 1 vs. 3 conditions) and 2 (2 vs. 3 and 2 vs. 4 conditions) pellets for each rearing condition. The SC rats displayed the largest relative response rate on the 
small lever for both magnitudes. An ANOVA revealed a significant effect of reward magnitude, $F(1,21)=15.77, p=.001$, and a significant rearing condition effect, $F(2,21)=3.63, p=.044$, but no interaction, $F(2,21)=2.13, p=.144$. The rearing condition effect was due to greater relative response rates in the SC group compared to the IC group, with the EC group intermediate between the two.

Additional analyses were conducted using one-sample $t$-tests to assess whether the relative response rates on the small lever were different from 1.0 (no change). Examining the change in rate in the SM-1 conditions provides an index of generalization from the large lever to the small lever. Because the SM-1 reward was the same as in the baseline phase, there should be no basis for response rates to increase apart from generalization from the large lever. An examination of the SM-1 responding for each rearing condition revealed that the $\mathrm{EC}$ rats, $t(7)=$ $3.09, p=.018$, and SC rats, $t(7)=4.36, p=.003$, both showed significant generalization to the SM-1 reward. However, the IC rats did not generalize to the SM-1 reward, $t(7)=1.19, p=.273$. An examination of the response to the SM-2 reward revealed a similar pattern in that the EC rats, $t(7)=3.55, p=.009$, and SC rats, $t(7)=4.81, p=.002$, displayed increased responding, but the IC rats did not, $t(7)=1.61, p=.152$

The general pattern of results from the reward magnitude sensitivity test indicated that the IC rats discriminated between the small and large rewards more effectively by maintaining a low rate of response to the SM-1 and SM-2 rewards, whereas the EC and IC rats generalized to the small rewards to a greater degree. To assess this more directly, Figure 10 presents the absolute response rates to the small and large rewards for each of the four pairs of magnitudes in the reward sensitivity test for each rearing condition. As was apparent in the baseline phase, the IC rats responded at a higher rate than the EC and SC rats. In addition, all three groups showed 
generally higher rates to the large rewards than to the small rewards, although this was more apparent in the IC rats than in the other groups, and was weakest in the SC rats. An analysis of each group's discrimination of each of the reward pairs confirmed these general observations. The EC rats did not differ significantly in their response to the large versus the small reward for any of the magnitude pairs, largest $t(7)=1.43, p=.196$. The SC rats also did not differ in their response rates to the large versus small rewards, largest $t(7)=1.44, p=.192$. However, the IC rats did significantly discriminate the SM-1/LG-3 pair, $t(7)=3.57, p=.009$, and had a nearsignificant discrimination of the SM-1/LG-2 pair, $t(7)=2.25, p=.059$ and the SM-2/LG-3 pair, $t(7)=2.0, p=.082$. Thus, consistent with the reward contrast phase, and with the reward challenge task in Experiment 1, the IC rats significantly discriminated between the small and large rewards.

\section{General Discussion}

The present pair of experiments sought to assess locomotor activity, impulsive choice, impulsive action, reward discrimination, and reward sensitivity as a function of rearing environment. The locomotor activity results in both experiments indicated that isolated rearing resulted in significant increases in locomotor activity, whereas enrichment either slightly decreased (Figure 1) or maintained (Figure 5) the pre-rearing activity levels. In Experiment 2, an additional assessment of the standard rearing condition was conducted, which showed similar findings to the effects to isolated rearing as has previously been reported (Cain, et al., 2012; Gill, Arnold, \& Cain, 2012; Wooters, et al., 2011). Experiment 2 also assessed locomotor activity following the reward sensitivity testing under saline and d-amphetamine. These additional assessments indicated that the rearing effects on locomotor activity were maintained over the course of the study, and that the differences among the rearing conditions were still present under 
d-amphetamine exposure. As a whole, the locomotor activity results are consistent with previous reports in the literature suggesting that enrichment decreases general locomotor activity (Bowling, et al., 1993; Lore \& Levowitz, 1966; Simpson \& Kelly, 2011), and that EC rats display reduced locomotor activity while under the influence of AMP in comparison to IC rats (Bardo, et al., 1995; Bardo \& Dwoskin, 2004; Cain, et al., 2012).

With regard to impulsive choice behavior, IC rats tended to choose the LL outcome less often than the EC rats (Figure 2), but this difference was not significant. However, the latencies to initiate forced choice trials (Shapiro, et al., 2008) by the IC and EC rats indicated that the IC rats exhibited shorter latencies to initiate the SS forced choice trial than they did for the LL forced choice trials, suggesting that they valued the SS option more than the LL option; the EC rats, however, displayed similar valuation of the two options on forced choice trials. Thus, in considering the forced choice latencies, the present pattern of results is generally consistent with previous reports indicating that enrichment decreases the degree of impulsive choice behavior (Perry, et al., 2008), but further research will be needed to verify these differences where we did not observe significant effects of rearing condition on impulsive choice behavior.

In the impulsive action task (assessed by the DRL task), the IC rats displayed more efficient performance, particularly on the DRL 30-s schedule, indicating decreased impulsive action compared to the EC rats. These findings are consistent with several recent reports of greater impulsive action in EC rats (Dalley, et al., 2002; Hill, et al., 2012; Zeeb, et al., 2012). However, a much earlier report by Ough et al. (1972) indicated that IC rats were less efficient on a DRL task. Thus, given the discrepancies in the literature, it seems that further research is warranted to determine the factors that are involved in determining the effects of rearing conditions on performance in both impulsive choice and impulsive action tasks. 
One possible common mechanism that may explain the pattern of effects is differences in the more fundamental mechanism of reward magnitude sensitivity (see Brunner \& Hen, 1997; Ho, et al., 1998). The results of the reward challenge phase in Experiment 1 indicated that the IC rats were more sensitive to differences in reward magnitude when the delays to reward were equal for the SS and LL choices (Figures 2 and 3). This finding was mirrored in Experiment 2 in the reward contrast and magnitude sensitivity tests in which the IC rats displayed more selective responding to the large reward whereas the EC and SC rats generalized to the small reward lever. This finding, coupled with the higher overall response rates in the IC rats (Figures 6 and 10) suggests that the IC rats were more sensitive to the reinforcing effects of the food reward, displaying greater incentive motivation to work for reward and greater sensitivity to magnitude differences. This difference could explain the more efficient performance on the DRL schedule by the IC rats as they were presumably more motivated to earn rewards than the EC rats. In the impulsive choice task, although there were no significant differences in choice behavior, the IC rats did display differences in their forced choice latencies, again suggesting that the IC rats better discriminated the two outcomes. In addition, the shorter latencies for the SS outcome suggest that the IC rats may value rewards that result in higher local rates of reward earning, at least in cases where the difference in magnitude of the two options is not substantial. With the selected parameters for the impulsive choice task, the SS option did result in a higher local rate of reinforcement (1 pellet in 10 s vs. 2 pellets in 30 s), whereas the LL option resulted in a higher global reinforcement rate due to the fixed 60-s ITI (so that the SS resulted in 1 pellets in $70 \mathrm{~s}$ and the LL in 2 pellets in 90 s). Future research examining choice over multiple delay options in the EC and IC rats should provide some deeper understanding of their patterns of choice behavior in relation to the local versus global maximization of reward. 
The present findings are consistent with several previously published studies examining differences in reward sensitivity and incentive motivation as a function of rearing environment. For example, the higher overall response rates in IC rats when working for food (Figures 6 and 10) is consistent with previous reports of increased responses for a novel visual stimulus (Cain, et al., 2006) and for sucrose reward under deprivation in IC rats (Gill \& Cain, 2010; Wood, et al., 2006). Previous research has also suggested that enriched rats are less sensitive to reward than group-housed non-enriched rats (van den Harst, Baars, \& Spruijt, 2003), consistent with the poorer reward discrimination evidenced in both experiments (Figures 2, 3, and 10). Thus, the suggested differential-rearing-induced differences in impulsivity may be explained by a more parsimonious mechanism of reward sensitivity. Overall, it appears that IC rats may be characterized by augmented reward sensitivity and incentive motivation (Beckmann \& Bardo, 2012; Fone \& Porkess, 2008; Lapiz et al., 2003), which may contribute to their behaviors in locomotor activity, impulsive choice and impulsive action tasks.

The differences observed in reward sensitivity between EC and IC rats are likely due to both the structural and functional differences caused by differential rearing in brain regions associated with reward processing. For example, enrichment alters both the structure and function of the nucleus accumbens (Bardo et al., 1999; Rahman \& Bardo, 2008; Wood, Buse, Wellman, \& Rebec, 2005; Wood \& Rebec, 2009), a brain region important for reward processing and incentive motivation, and that appears to play a particular role in integrating reward amount and delay information (Cardinal, Pennicott, Sugathapala, Robbins, \& Everitt, 2001; Galtress \& Kirkpatrick, 2010; Ikemoto \& Panksepp, 1999). The observed differences in reward sensitivity likely contribute to the differences observed in impulsive choice and impulsive action observed in the current study. Additionally, differential rearing alters both the serotonergic and 
dopaminergic systems within the PFC; isolation rearing decreases serotonin levels and dopamine function when compared to enriched rearing (Brenes, et al., 2008; Zhu, et al., 2005). Decreases in serotonergic and dopaminergic function in the PFC have been linked to increases in impulsive choice behavior (Ho, et al., 1998; Loos et al., 2010; Pardey, et al., 2012). Therefore, these results suggest that rearing-induced changes in the dopaminergic and serotonergic systems may contribute to the differences observed between EC and IC rats in impulsive choice behavior. The present results suggest that an important trajectory of future research may be to elucidate the mechanisms of the effects of differential rearing on incentive motivation, reward valuation, and sensitivity to reward amounts which may be exerting an impact on behavior in impulsivity tasks. 


\section{Acknowledgements}

The authors would like to thank Jennifer Arnold, Bethany Caldwell, Angela Crumer, Ana Garcia, Diedrian Walker and Mayabeth Jagosz for their assistance with the conduct of the studies. The research was supported by a University Research Small Grant (2598) from Kansas State University, and partially supported by an NIMH grant (085739) awarded to Kansas State University. 


\section{References}

Barbelivien, A., Billy, E., Lazarus, C., Kelche, C., \& Majchrzak, M. (2008). Rats with different profiles of impulsive choice behavior exhibit differences in responses to caffeine and $d$ amphetamine and in medial prefrontal cortex 5-HT utilization. Behavioural Brain Research, 187, 273-283. doi: 10.1016/j.bbr.2007.09.020

Bardo, M. T., Bowling, S. L., Rowlett, J. K., Manderscheid, P., Buxton, S. T., \& Dwoskin, L. P. (1995). Environmental enrichment attenuates locomotor sensitization, but not in vitro dopamine release, induced by amphetamine. Pharmacology Biochemistry and Behavior, 51, 397-405. doi: 10.1016/0091-3057(94)00413-D

Bardo, M. T., \& Dwoskin, L. P. (2004). Biological connection between novelty- and drugseeking motivational systems. Nebraska Symposium on Motivation, 50, 127-158.

Bardo, M. T., Valone, J., Robinet, P. M., Buxton, S. T., Shaw, W., \& Dwoskin, L. P. (1999). Environmental enrichment enhances the stimulant effect of intravenous amphetamine: Search for a cellular mechanism in the nucleus accumbens. Psychobiology, 27, 292-299.

Bari, A., Theobald, D. E., Caprioli, D., Mar, A. C., Aidoo-Micah, A., Dalley, J. W., et al. (2010). Serotonin modulates sensitivity to reward and negative feedback in a probabilistic reversal learning task in rats. Neuropsychopharmacology, 35, 1290-1301. doi: 10.1038/npp.2009.233

Bateson, M., \& Kacelnik, A. (1995). Preferences for fixed and variable food sources: variability in amount and delay. Journal of the Experimental Analysis of Behavior, 63(3), 313-329. doi: 10.1901/jeab.1995.63-313 
Beckmann, J. S., \& Bardo, M. T. (2012). Environmental enrichment reduces attribution of incentive salience to a food-associated stimulus. Behavioural Brain Research, 226, 331334. doi: 10.1016/j.bbr.2011.09.021

Bowling, S. L., Rowlett, J. K., \& Bardo, M. T. (1993). The effect of environmental enrichment on amphetamine-stimulated locomotor activity, dopamine synthesis and dopamine release. Neuropharmacology, 32, 885-893. doi: 10.1016/0028-3908(93)90144-R

Brenes, J. C., \& Fornaguera, J. (2008). Effects of environmental enrichment and social isolation on sucrose consumption and preference: associations with depressive-like behavior and ventral striatum dopamine. Neuroscience Letters, 436, 278-282. doi: 10.1016/j.neulet.2008.03.045

Brenes, J. C., Rodríguez, O., \& Fornaguera, J. (2008). Differential effect of environment enrichment and social isolation on depressive-like behavior, spontaneous activity and serotonin and norepinephrine concentration in prefrontal cortex and ventral striatum Pharmacology, Biochemistry and Behavior, 89, 85-93. doi: 10.1016/j.pbb.2007.11.004

Brunner, D., \& Hen, R. (1997). Insights into the neurobiology of impulsive behavior from serotonin receptor knockout mice. Annals of the New York Academy of Sciences, 836, 81105. doi: 10.1111/j.1749-6632.1997.tb52356.x

Cain, M. E., Green, T. A., \& Bardo, M. T. (2006). Environmental enrichment decreases responding for visual novelty. Behavioural Processes, 73, 360-366. doi: 10.1016/j.beproc.2006.08.007

Cain, M. E., Mersmann, M. G., Gill, M. J., \& Pittenger, S. T. (2012). Dose dependent effects of differential rearing on amphetamine-induced hyperactivity. Behavioural Pharmacology, 23(8), 744-753. doi: 10.1097/FBP.0b013e32835a38ec 
Cardinal, R. N., Pennicott, D. R., Sugathapala, C. L., Robbins, T. W., \& Everitt, B. J. (2001). Impulsive choice induced in rats by lesions of the nucleus accumbens core. Science, 292, 2499-2501.

Catania, A. C. (1970). Reinforcement schedules and psychophysical judgments. In W. N. Schoenfeld (Ed.), Theory of Reinforcement Schedules (pp. 1-42). New York: AppletonCentury-Crofts.

Coolon, R. A., \& Cain, M. E. (2009). Effects of mecamylamine on nicotine-induced conditioned hyperactivity and sensitization in differentially reared rats. Pharmacology Biochemistry and Behavior, 93, 59-66. doi: 10.1016/j.pbb.2009.04.007

Dalley, J. W., Theobald, D. E., Pereira, E. A. C., Li, P. M. M. C., \& Robbins, T. W. (2002). Specific abnormalities in serotonin release in the prefrontal cortex of isolation-reared rats measured during behavioural performance of a task assessing visuospatial attention and impulsivity. Psychopharmacology, 164, 329-340.

Deehan, G. A., Cain, M. E., \& Kiefer, S. W. (2007). Differential rearing conditions alter operant responding for ethanol in outbred rats. Alcoholism: Clinical and Experimental Research, 31, 1692-1698. doi: 10.1111/j.1530-0277.2007.00466.x

Deehan, G. A., Palmatier, M. I., Cain, M. E., \& Kiefer, S. W. (2011). Differential rearing conditions and alcohol-preferring rats: consumption of and operant responding for ethanol. Behavioral Neuroscience, 125, 184-193. doi: 10.1037/a0022627

Diergaarde, L., Pattij, T., Poortvliet, I., Hogenboom, F., de Vries, W., Schoffelmeer, A. N. M., et al. (2008). Impulsive choice and impulsive action predict vulnerability to distinct stages of nicotine seeking in rats. Biological Psychiatry, 63, 301-308. doi:

10.1016/j.biopsych.2007.07.011 
Evenden, J. L. (1999). Varieties of impulsivity. Psychopharmacology, 146, 348-361. doi: 10.1007/PL00005481

Fone, K. C. F., \& Porkess, M. V. (2008). Behavioural and neurochemical effects of post-weaning social isolation in rodents - relevance to development neuropsychiatric disorders. Neuroscience and Biobehavioral Reviews, 32, 1087-1102. doi: 10.1016/j.neubiorev.2008.03.003

Galtress, T., Garcia, A., \& Kirkpatrick, K. (2012). Individual differences in impulsive choice and timing in rats. Journal of the Experimental Analysis of Behavior, 98(1), 65-87.

Galtress, T., \& Kirkpatrick, K. (2010). The role of the nucleus accumbens core in impulsive choice, timing, and reward processing. Behavioral Neuroscience, 124, 26-43. doi: 10.1037/a0018464

Garcia, A., \& Kirkpatrick, K. (2013). Impulsive choice behavior in four strains of rats: Evaluation of possible models of Attention-Deficit/Hyperactivity Disorder. Behavioural Brain Research, 238, 10-22.

Gill, M. J., Arnold, J. C., \& Cain, M. E. (2012). Impact of mGluR5 during amphetamine-induced hyperactivity and conditioned hyperactivity in differentially reared rats. Psychopharmacology, 221, 227-237. doi: 10.1007/s00213-011-2565-0

Gill, M. J., \& Cain, M. E. (2010). Effects of satiety on operant responding in rats raised in enrichment. Behavioral Pharmacology, 22, 40-48. doi: 10.1097/FBP.0b013e3283425a86

Green, L., \& Estle, S. J. (2003). Preference reversals with food and water reinforcers in rats. Journal of the Experimental Analysis of Behavior, 79, 233-242. doi: 10.1901/jeab.2003.79-233 
Green, T. A., Gehrke, B. J., \& Bardo, M. T. (2002). Environmental enrichment decreases intravenous amphetamine self- administration in rats: dose-response functions for fixedand progressive-ratio schedules. Psychopharmacology, 162, 373-378. doi: 10.1007/s00213-002-1134-y

Hellemans, K. G. C., Nobrega, J. N., \& Olmstead, M. C. (2005). Early environmental experience alters baseline and ethanol-induced cognitive impulsivity: relationship to forebrain 5$\mathrm{HT}_{1 \mathrm{~A}}$ receptor binding. Behavioural Brain Research, 159, 207-220. doi: 10.1016/j.bbr.2004.10.018

Hill, J. C., Covarrubias, P., Terry, J., \& Sanabria, F. (2012). The effect of methylphenidate and rearing environment on behavioral inhibition in adult male rats. Psychopharmacology, 219, 353-362. doi: 10.1007/s00213-011-2552-5

Ho, M.-Y., Al-Zahrani, S. S. A., Al-Ruwaitea, A. S. A., Bradshaw, C. M., \& Szabadi, E. (1998). 5-hydroxytryptamine and impulse control: prospects for a behavioural analysis. Journal of Psychopharmacology, 12, 68-78. doi: 10.1177/026988119801200109

Ikemoto, S., \& Panksepp, J. (1999). The role of nucleus accumbens dopamine in motivated behavior: a unifying interpretation with special reference to reward-seeking. Brain Research Reviews, 31, 6-41. doi: 10.1016/S0165-0173(99)00023-5

Jones, G. H., Marsden, C. A., \& Robbins, T. W. (1990). Increased sensitivity to amphetamine and reward-related stimuli following social isolation in rats: possible disruption of dopamine-dependent mechanisms of the nucleus accumbens. Psychopharmacology, 102, 364-372. doi: 10.1007/BF02244105 
Kacelnik, A., Vasconcelos, M., Monteiro, T., \& Aw, J. (2011). Darwin's "tug-of-war" vs. starlings' "horse-racing": how adaptations for sequential encounters drive simultaneous choice. Behavioral Ecology and Sociobiology, 65, 547-558.

Lapiz, M. D. S., Fulford, A., Muchimapura, S., Mason, R., Parker, T., \& Marsden, C. A. (2003). Influence of postweaning social isolation in the rat on brain development, conditioned behavior, and neurotransmission. Neuroscience and Behavioral Physiology, 33, 13-29. doi: 10.1023/A:1021171129766

Loos, M., Pattij, T., Janssen, M. C., Counotte, D. S., Schoffelmeer, A. N., Smit, A. B., et al. (2010). Dopamine receptor D1/D5 gene expression in the medial prefrontal cortex predicts impulsive choice in rats. Cerebral Cortex, 20, 1064-1070. doi:

10.1093/cercor/bhp167

Lore, R. K., \& Levowitz, A. (1966). Differential rearing and free versus forced exploration. Psychonomic Science, 5, 421-422.

Morgan, M., \& Einon, D. (1975). Incentive motivation and behavioral inhibition in sociallyisolated rats. Physiology \& Behavior, 15, 405-409. doi: 10.1016/0031-9384(75)90250-4

Odum, A. L. (2011). Delay discounting: I'm a k, you're a k. Journal of the Experimental Analysis of Behavior, 96(3), 427-439.

Ough, B. R., Beatty, W. W., \& Khalili, J. (1972). Effects of isolated and enriched rearing on response inhibition. Psychonomic Science, 27, 293-294.

Pardey, M. C., Kumar, N. N., Goodchild, A. K., \& Cornish, J. L. (2012). Catecholamine receptors differentially mediate impulsive choice in the medial prefrontal and orbitofrontal cortex. Journal of Psychopharmacology. doi: 0269881112465497 
Perry, J. L., Stairs, D. J., \& Bardo, M. T. (2008). Impulsive choice and environmental enrichment: effects of d-amphetamine and methylphenidate. Behavioural Brain Research, 193, 48-54. doi: 10.1016/j.bbr.2008.04.019

Rahman, S., \& Bardo, M. T. (2008). Environmental enrichment increases amphetamine-induced glutamate neurotransmission in the nucleus accumbens: a neurochemical study. Brain Research, 1197, 40-46. doi: 10.1016/j.brainres.2007.12.052

Reboreda, J. C., \& Kacelnik, A. (1991). Risk sensitivity in starlings: variability in food amount and delay. Behavioral Ecology, 2(4), 301-308.

Renner, M. J., \& Rosenzweig, M. R. (1987). Enriched and impoverished environments: Effects on brain and behavior. New York: Springer-Verlag.

Roberts, S. (1981). Isolation of an internal clock. Journal of Experimental Psychology: Animal Behavior Processes, 7, 242-268. doi: 10.1037/0097-7403.7.3.242

Roesch, M. R., Calu, D. J., \& Schoenbaum, G. (2007). Dopamine neurons encode the better option in rats deciding between differently delayed or sized rewards. Nature Neuroscience, 10(12), 1615-1624.

Shapiro, M. S., Siller, S., \& Kacelnik, A. (2008). Simultaneous and sequential choice as a function of reward delay and magnitude: normative, descriptive and process-based models tested in the European starling (Sturnus vulgaris). Journal of Experimental Psychology: Animal Behavior Processes, 34, 75-93. doi: 10.1037/0097-7403.34.1.75

Simpson, J., \& Kelly, J. P. (2011). The impact of environmental enrichment in laboratory ratsbehavioural and neurochemical aspects. Behavioural Brain Research, 222, 246-264. doi: 10.1016/j.bbr.2011.04.002 
Smith, J. K., Neill, J. C., \& Costall, B. (1997). Post-weaning housing conditions influence the behavioural effects of cocaine and $d$-amphetamine. Psychopharmacology, 131, 23-33. doi: $10.1007 / s 002130050261$

Smith, M. A., Bryant, P. A., \& McClean, J. M. (2003). Social and environmental enrichment enhances sensitivity to the effects of kappa opioids: studies on antinociception, diuresis and conditioned place preference. Pharmacology, Biochemistry and Behavior, 76, 93101. doi: 10.1016/S0091-3057(03)00189-8

Smith, M. A., Chisholm, K. A., Bryant, P. A., Greene, J. L., McClean, J. M., Stoops, W. W., et al. (2005). Social and environmental influences on opioid sensitivity in rats: importance of an opioid's relative efficacy at the mu-receptor. Psychopharmacology, 181, 27-37. doi: $10.1007 / \mathrm{s} 00213-005-2218-2$

Stairs, D. J., \& Bardo, M. T. (2009). Neurobehavioral effects of environmental enrichment and drug abuse vulnerability. Pharmacology Biochemistry and Behavior, 92, 377-382. doi: 10.1016/j.pbb.2009.01.016

Tatham, T. A., \& Zurn, K. R. (1989). The MED-PC experimental apparatus programming system. Behavior Research Methods, Instruments, \& Computers, 21, 294-302. doi: 10.3758/BF03205598

van den Harst, J. E., Baars, A.-M., \& Spruijt, B. M. (2003). Standard housed rats are more sensitive to rewards than enriched house rats as reflected by their anticipatory behaviour. Behavioural Brain Research, 142, 151-156. doi: 10.1016/S0166-4328(02)00403-5

Wood, D. A., Buse, J. E., Wellman, C. L., \& Rebec, G. V. (2005). Differential environmental exposure alters NMDA but not AMPA receptor subunit expression in nucleus accumbens core and shell. Brain Research, 1042, 176-183. doi: 10.1016/j.brainres.2005.02.029 
Wood, D. A., \& Rebec, G. V. (2009). Environmental enrichment alters neuronal processing in the nucleus accumbens core during appetitive conditioning. Brain Research, 1259, 59-67. doi: 10.1016/j.brainres.2008.12.038

Wood, D. A., Siegel, A. K., \& Rebec, G. V. (2006). Environmental enrichment reduces impulsivity during appetitive conditioning. Physiology \& Behavior, 88, 132-137. doi: 10.1016/j.physbeh.2006.03.024

Wooters, T. E., Bardo, M. T., Dwoskin, L. P., Midde, N. M., Gomez, A. M., Mactutus, C. F., et al. (2011). Effect of environmental enrichment on methylphenidate-induced locomotion and dopamine transporter dynamics. Behavioural Brain Research, 219, 98-107. doi: 10.1016/j.bbr.2011.01.001

Zeeb, F. D., Wong, A. C., \& Winstanley, C. A. (2012). Differential effects of environmental enrichment, social-housing, and isolation-rearing on a rat gambling task: dissociations between impulsive action and risky decision-making. Psychopharmacology, 1-15. doi: 10.1007/s00213-012-2822-X

Zhu, J., Apparsundaram, S., Bardo, M. T., \& Dwoskin, L. P. (2005). Environmental enrichment decreases cell surface expression of the dopamine transporter in rat medial prefrontal cortex. Journal of Neurochemistry, 93, 1434-1443. doi: 10.1111/j.14714159.2005.03130.x

Zimmermann, A., Stauffacher, M., Langhans, W., \& Würbel, H. (2001). Enrichment-dependent differences in novelty exploration in rats can be explained by habituation. Behavioural Brain Research, 121, 11-20. doi: 10.1016/S0166-4328(00)00377-6 


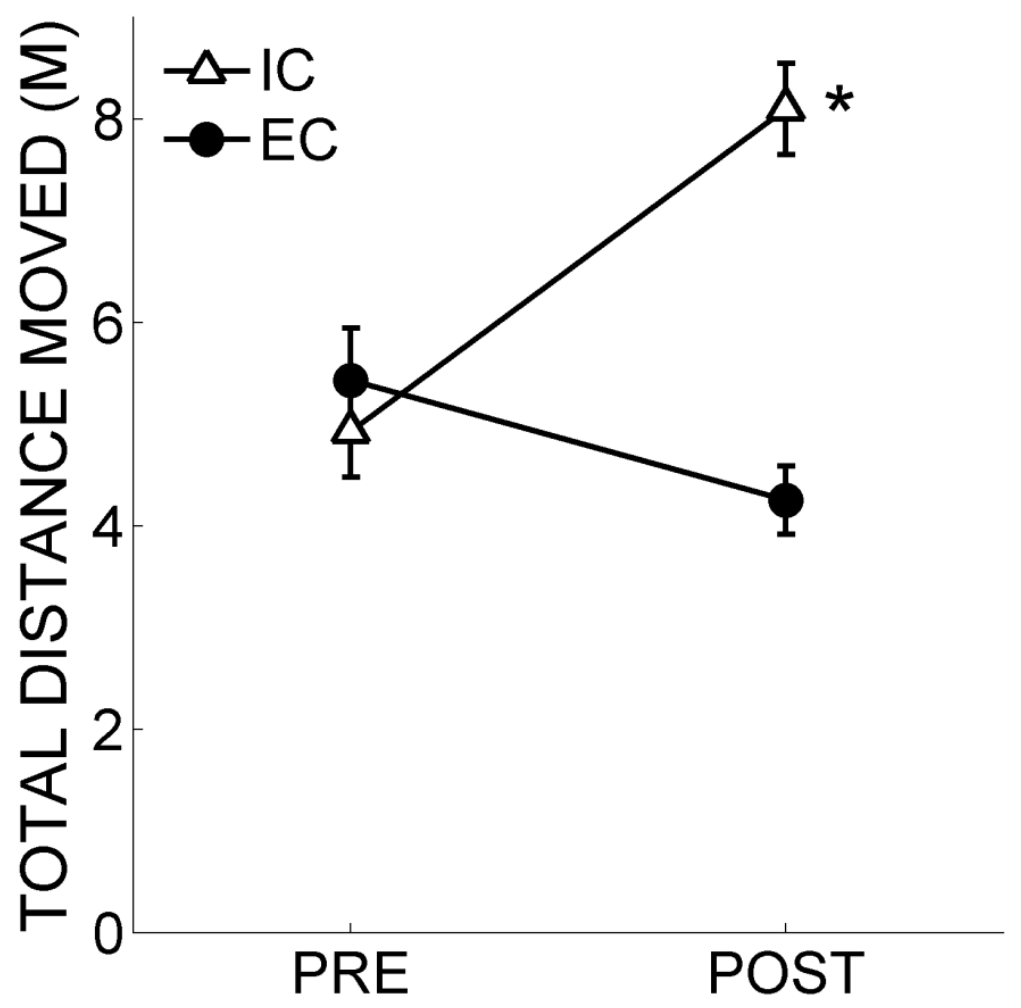

Figure 1. Total distance moved (m) by the isolated (IC) rats (white triangles) and enriched (EC) rats (black circles) in two locomotor screens - before the 30-day rearing period (PRE) and following the 30-day rearing period (POST) in Experiment 1. Asterisks mark significant group differences. 


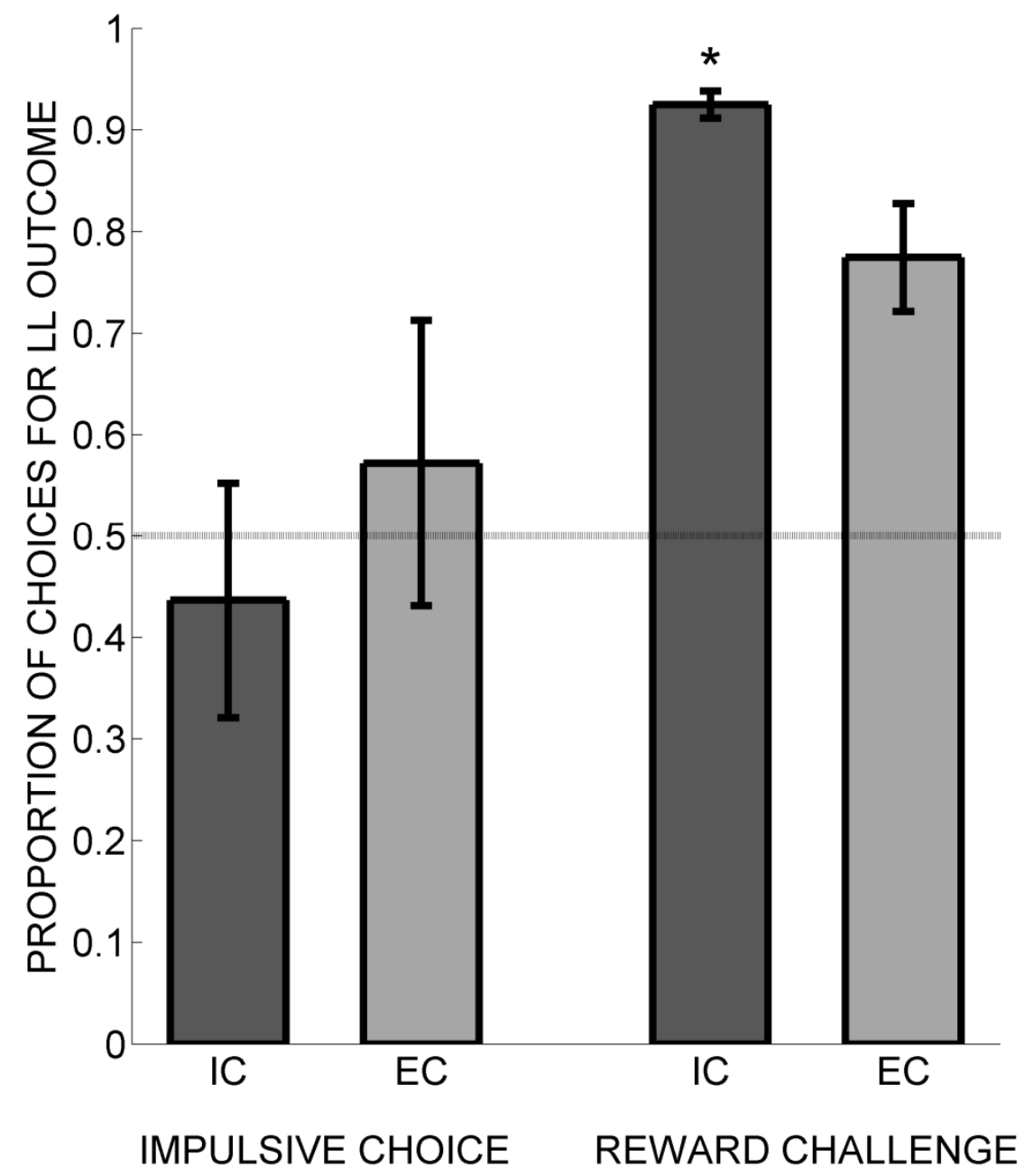

Figure 2. Proportion of choices for the larger-later (LL) outcome for isolated (IC) rats (darker bars) and enriched (EC) rats (lighter bars) in both phases of the impulsive-choice task delivered in Experiment 1. Asterisks mark significant group differences. 

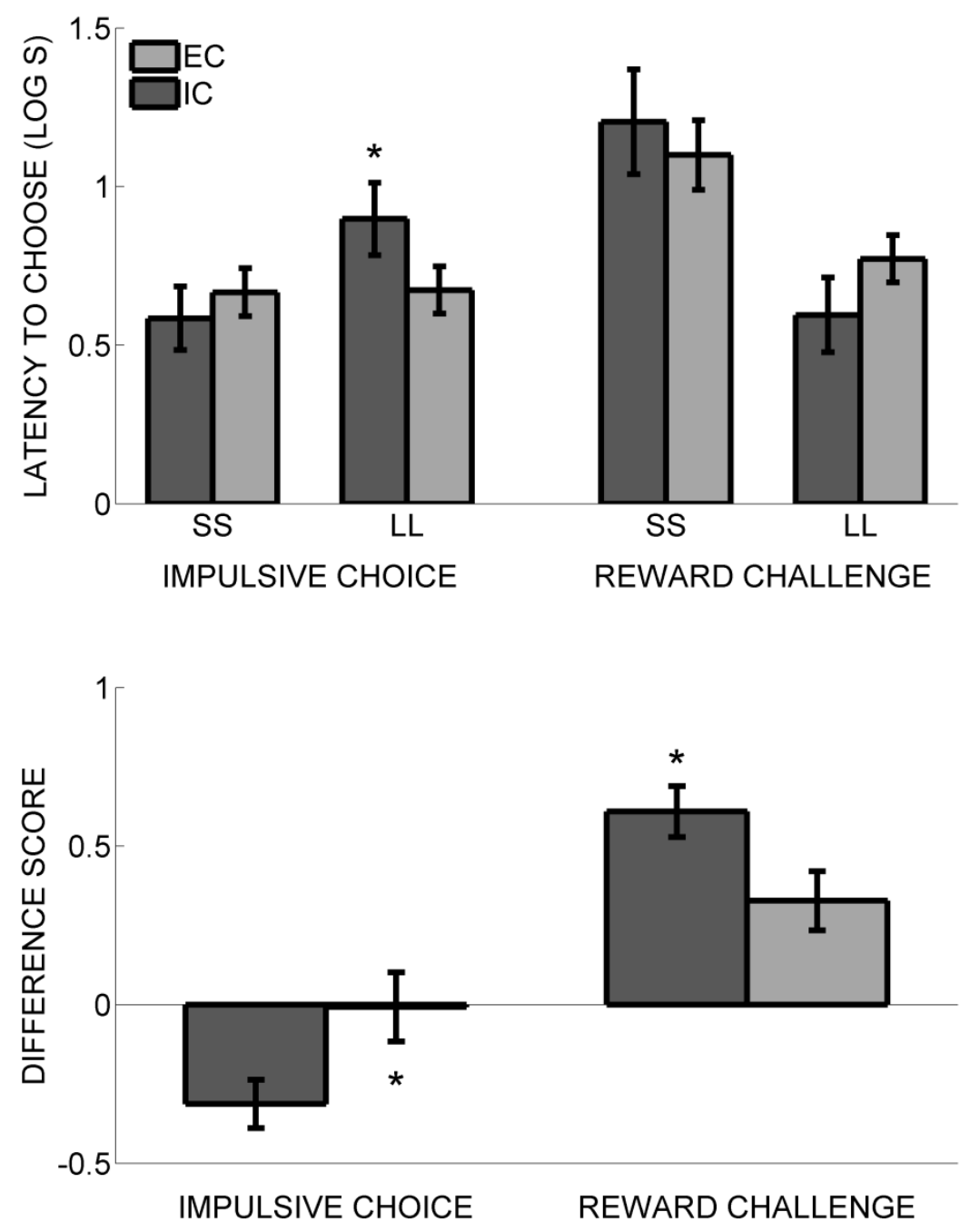

Figure 3. Top panel: Latency to choose (log s) the larger-later (LL) outcome and the smallersooner (SS) outcome in forced-choice trials for isolated (IC) rats (darker bars) and enriched (EC) rats (lighter bars). Asterisks mark significant group differences. Bottom panel: difference in SS and LL latencies for IC and EC rats. Data are taken from the impulsive choice and reward challenge tests in Experiment 1. Asterisks mark significant group differences. 


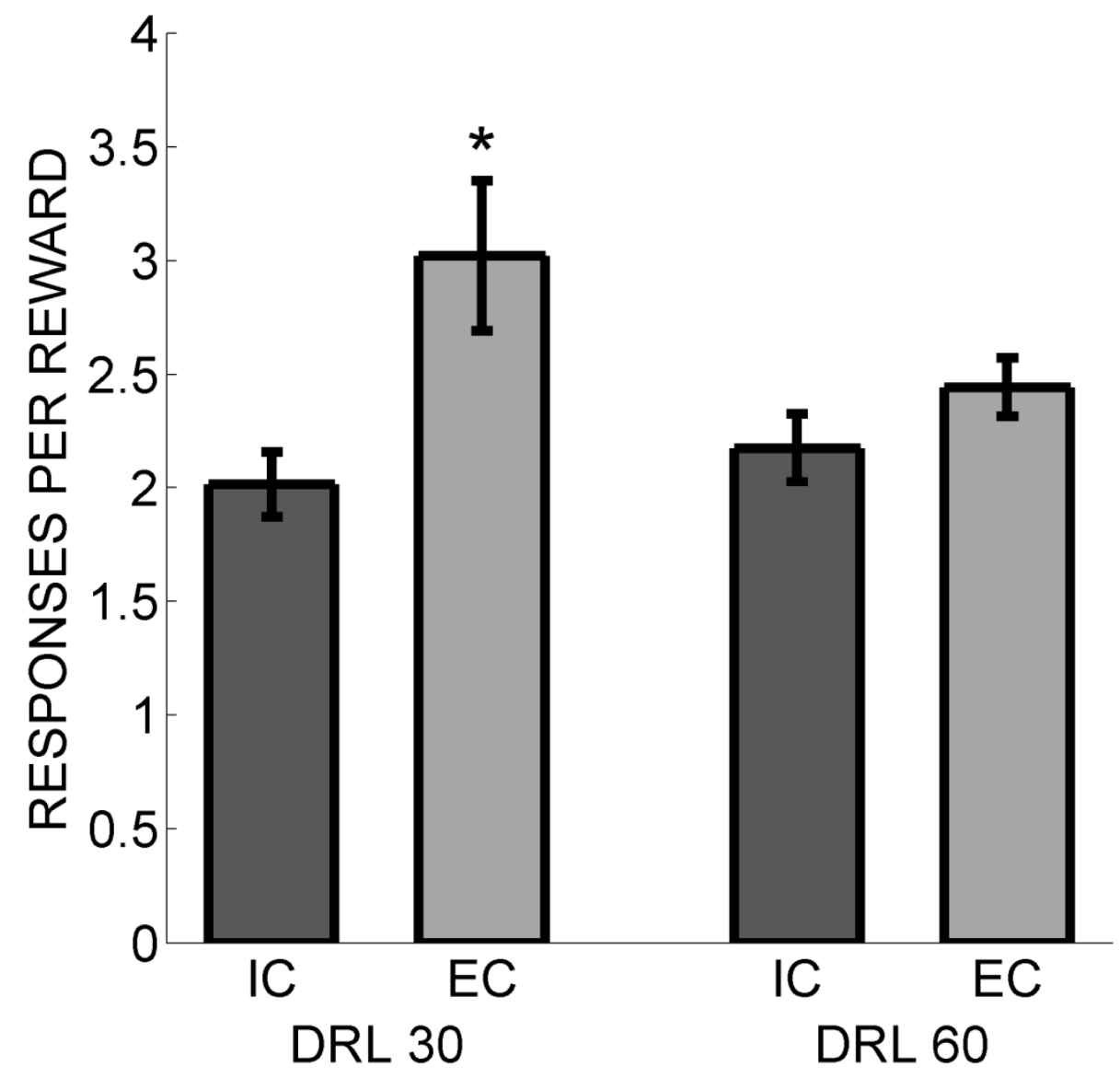

Figure 4. Number of operant responses per reward received for the isolated (IC) rats (darker bars) and the enriched (EC) rats (lighter bars) for both DRL criterions delivered in Experiment 1. DRL = differential reinforcement of low rate. Asterisks mark significant group differences. 


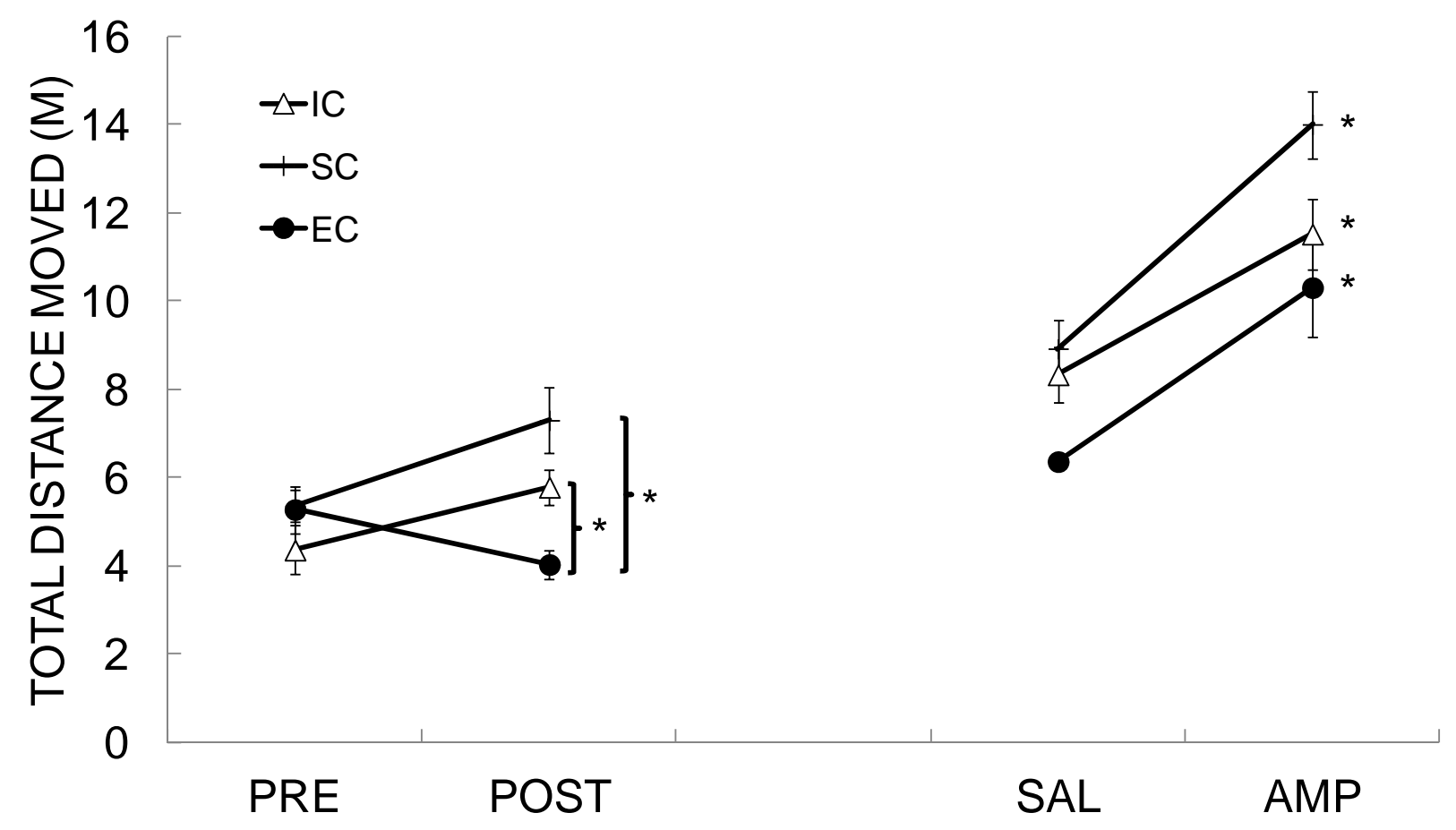

Figure 5. Total distance moved (m) during the 60-min locomotor activity test delivered prerearing (PRE), post-rearing (POST) and following the reward sensitivity testing while under saline (SAL) or d-amphetamine (AMP) in Experiment 2. Separate functions are presented for the isolated condition (IC), standard condition (SC) and enriched condition (EC) rats. Asterisks mark significant group differences in the POST phase and mark significant elevation in responding for each group in the AMP phase. 


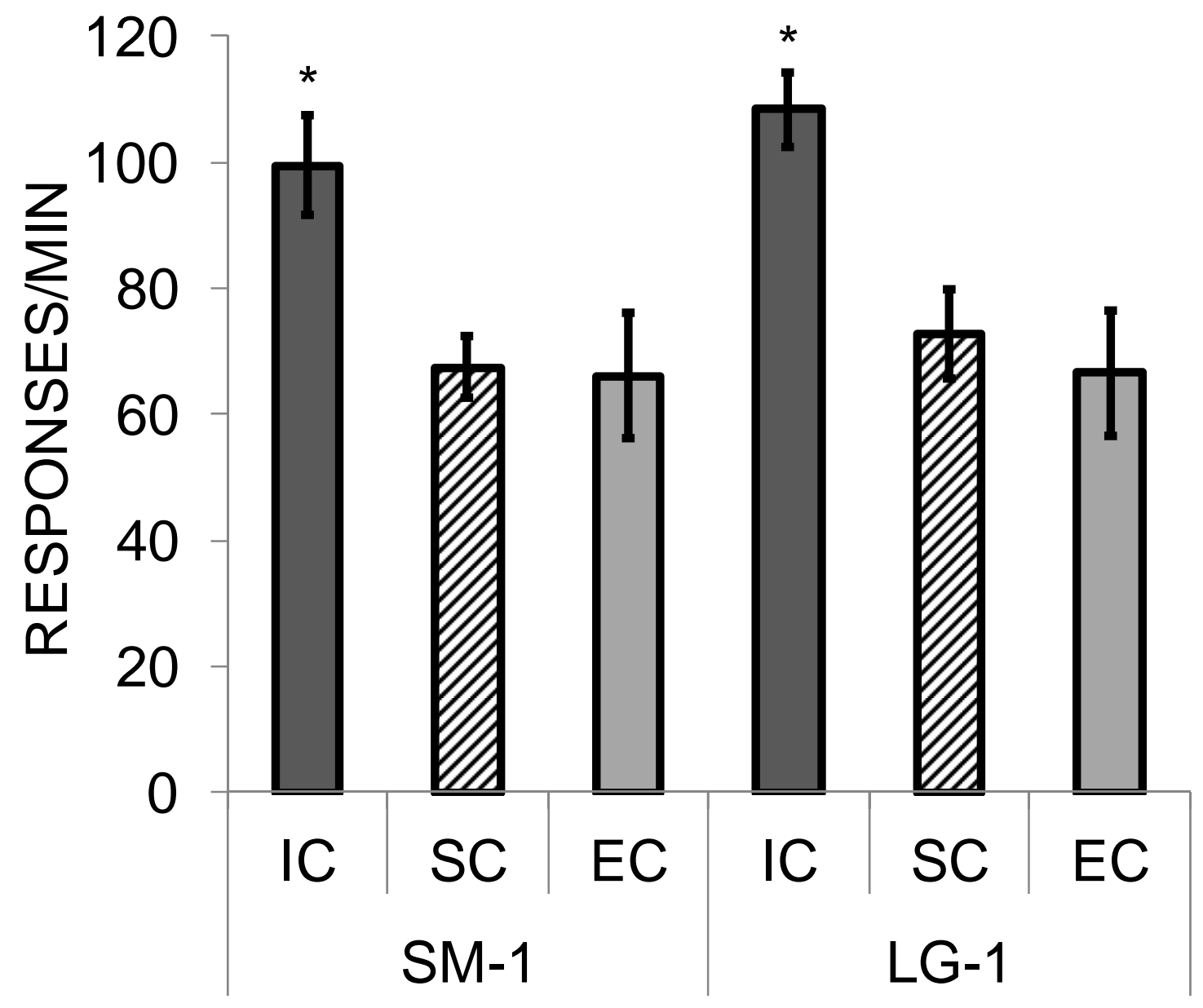

Figure 6. Mean ( \pm SEM) responses/min on the small (SM-1) and large (LG-1) during the baseline phase of the reward sensitivity test in Experiment 2, where only a single pellet was delivered on each lever. Separate data are presented for the isolated condition (IC), standard condition (SC) and enriched condition (EC) rats. Asterisks mark significant group differences. 


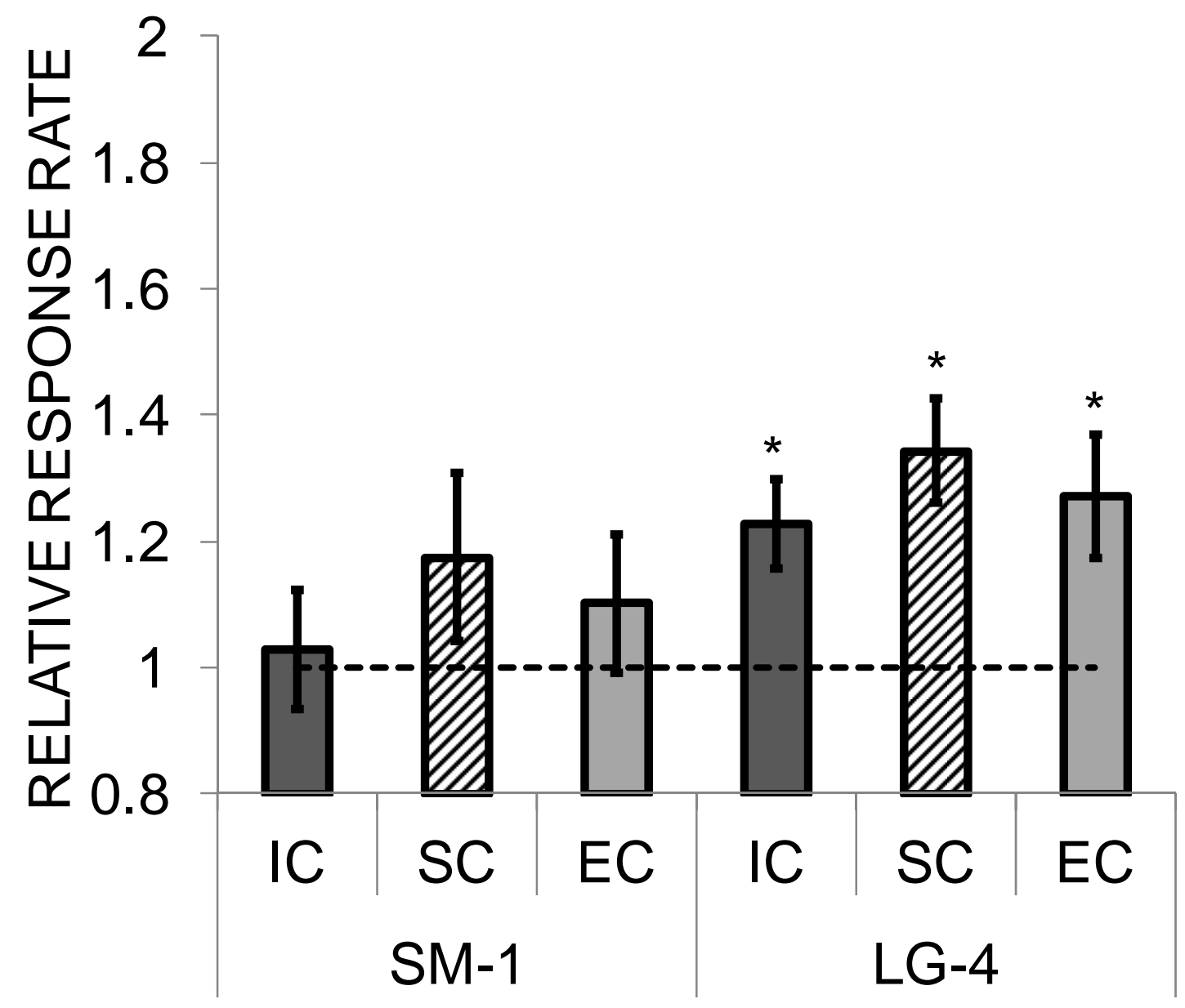

Figure 7. Mean ( \pm SEM) relative response rate on the small (SM-1) and large (LG-4) during the reward contrast sensitivity test in Experiment 2, where the magnitude on the large lever was increased to 4 pellets (while the small magnitude was maintained at 1 pellet). The relative response rate was the response rate on each lever during the contrast sensitivity test divided by the response rate on the same lever during the baseline phase. Separate data are presented for the isolated condition (IC), standard condition (SC) and enriched condition (EC) rats. Asterisks mark significant differences from 1.0. 


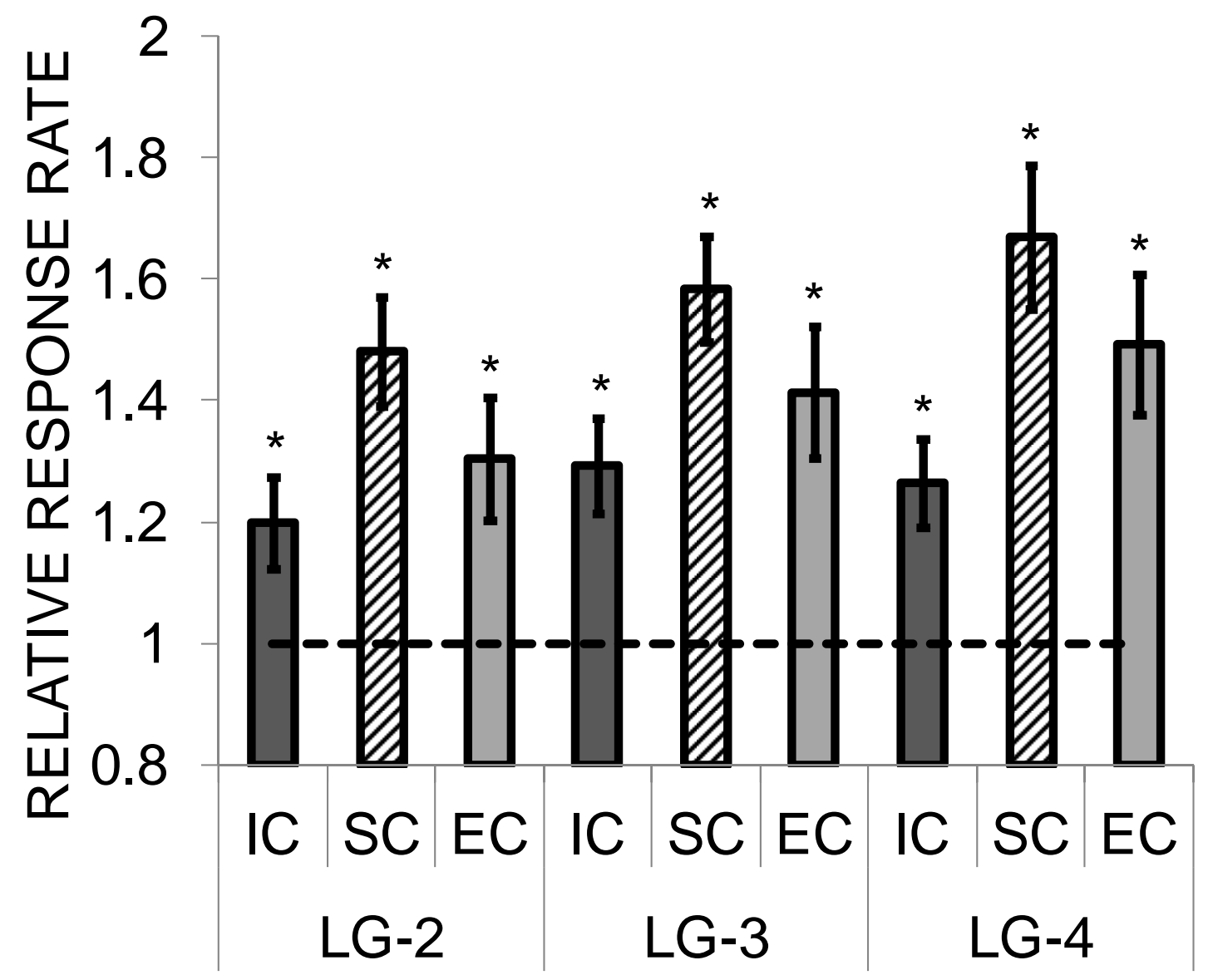

Figure 8. Mean ( \pm SEM) relative response rate on the large lever during the reward magnitude sensitivity test in Experiment 2 as a function of large reward magnitude. The relative response rate was determined by dividing the response rate on the large lever during each magnitude test by the response rate on the large lever during the baseline phase. Separate data are presented for the isolated condition (IC), standard condition (SC) and enriched condition (EC) rats. Asterisks mark significant differences from 1.0. 


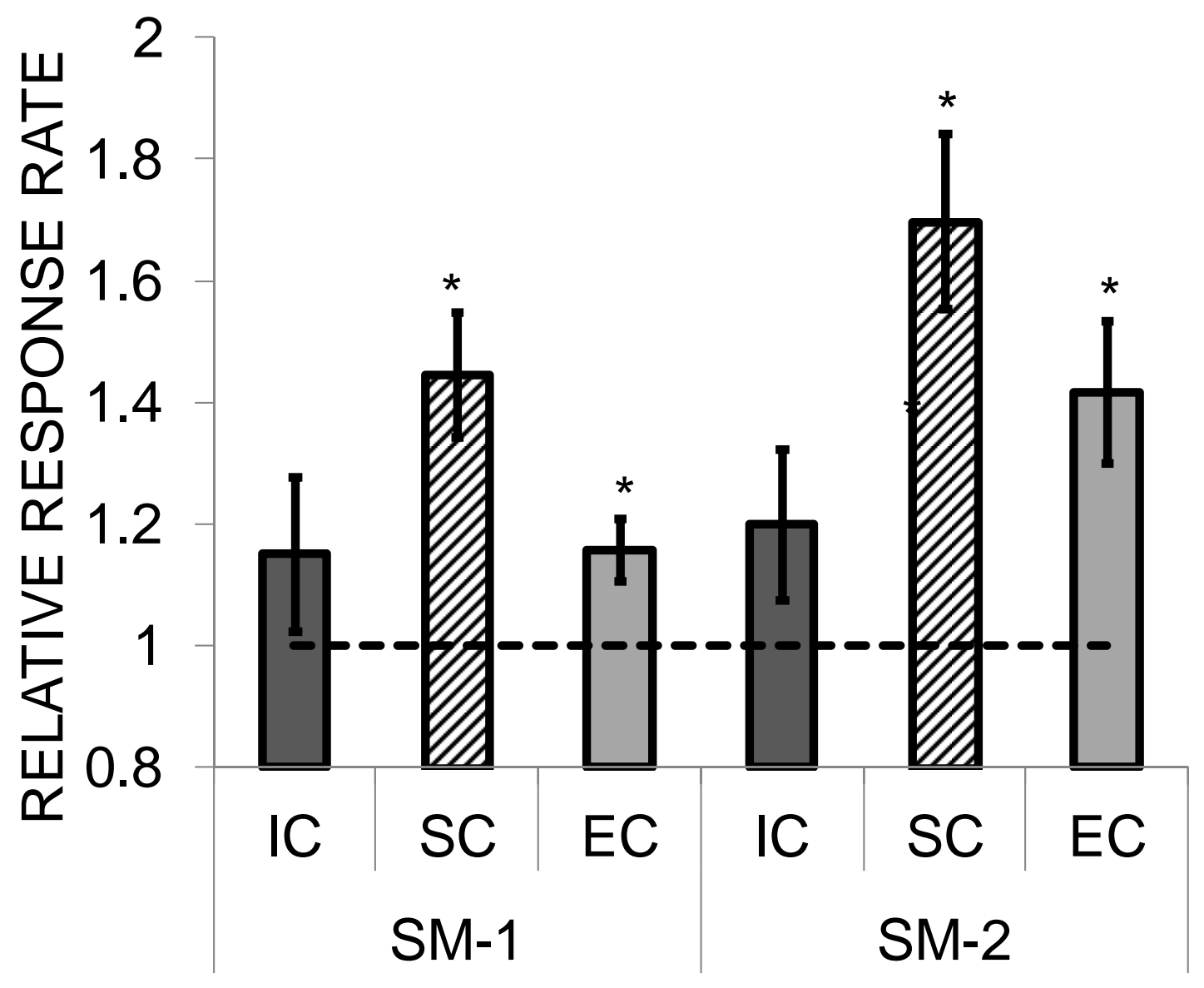

Figure 9. Mean ( \pm SEM) relative response rate on the small lever during the reward magnitude sensitivity test in Experiment 2 as a function of small reward magnitude. The relative response rate was determined by dividing the response rate on the small lever during each magnitude test by the response rate on the small lever during the baseline phase. Separate data are presented for the isolated condition (IC), standard condition (SC) and enriched condition (EC) rats. Asterisks mark significant differences from 1.0. 


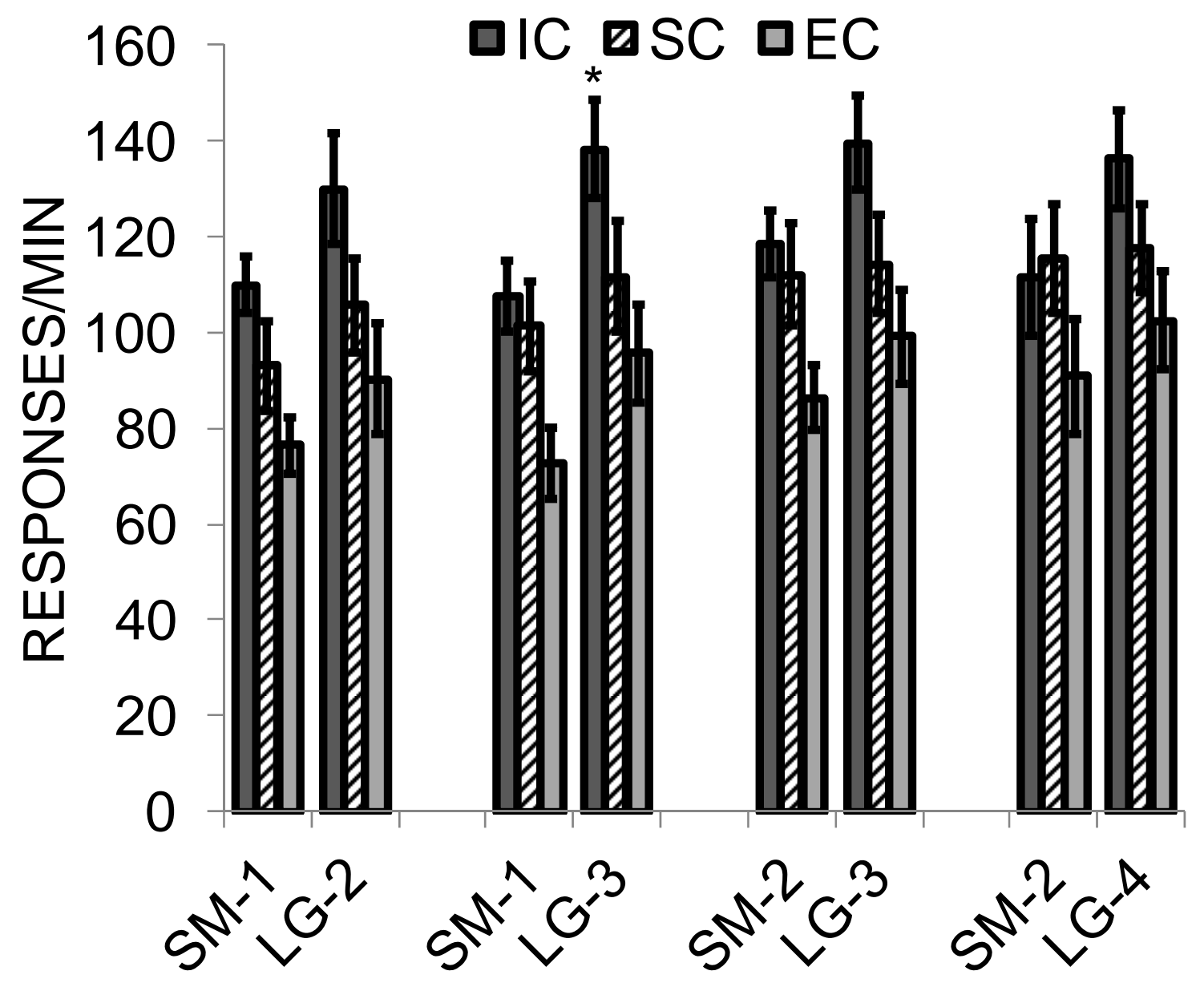

Figure 10. Mean ( \pm SEM) responses/min on the small and large lever during the reward magnitude sensitivity test in Experiment 2 as a function of small and large reward magnitude. Separate data are presented for each magnitude pair and for the isolated condition (IC), standard condition (SC) and enriched condition (EC) rats. Asterisks mark significant differences between levers. 